\title{
State-Space Dynamic Substructuring with the Transmission Simulator Method
}

\author{
Maren Scheel ${ }^{1} \cdot$ Mladen Gibanica $^{2,3} \cdot$ Anders Nord $^{4}$ \\ Received: 13 June 2018 / Accepted: 25 February 2019 / Published online: 28 March 2019 \\ (C) The Author(s) 2019
}

\begin{abstract}
Dynamic substructuring is a technique to simplify the analysis of complex structures. The vibrational problems of the constituent substructures are analysed and solved individually and their solutions are then assembled to form the global solution. In experimental dynamic substructuring, at least one of the constituent substructures is identified experimentally. The coupling interfaces are commonly simplified in such syntheses, which can result in poor prediction quality in many applications. The transmission simulator was introduced to address this problem. Transmission simulators are well-modelled parts attached to the interface of the substructures to be coupled. This allows for distributed interfaces and a relaxation of the coupling conditions by using the transmission simulator's analytical modes as a basis for the coupling equations, at the cost of adding a decoupling step to the substructuring problem. In this paper, the transmission simulator method is translated to the state-space substructuring domain. The methodology is applied to the Society for Experimental Mechanics' substructuring focus group's Ampair A600 test bed in form of experimental-analytical substructuring. The Ampair wind turbine's hub is used as the transmission simulator and is modelled with finite elements while the three blades, individually attached to the real hub, are experimentally identified. The three experimental blade hub systems are then coupled and two finite element hubs decoupled from the system, using the derived method. Finally, this system is compared to a directly measured hub with three blades by means of frequency response functions and modal properties.
\end{abstract}

Keywords Dynamic substructuring · Transmission simulator - State-space coupling · Experimental dynamics · Ampair wind turbine

\section{Introduction}

During the development of many complex products, such as those found in the automotive, aerospace and energy industries, dynamical properties must be assessed to prevent user discomfort or dangerous operation conditions. The finite element (FE) method [5] is commonly used to perform the necessary analyses. In many situations the full system under

Mladen Gibanica

mladen.gibanica@chalmers.se

1 Institute of Aircraft Propulsion Systems, University of Stuttgart, Pfaffenwaldring 6, 70569 Stuttgart, Germany

2 Department of Mechanics and Maritime Sciences, Chalmers University of Technology, Hörsalsvägen 7A, SE-412 96 Göteborg, Sweden

3 Volvo Car Corporation, SE-405 31 Göteborg, Sweden

4 Volvo Group Trucks Technology, SE-405 08 Göteborg, Sweden consideration is too complex to be analysed as a single component. In such situations dynamic substructuring techniques can be used. Many benefits follow from this strategy, such as simple parallelisation and efficient modelling of identical parts [16, 43]. Further, it allows combining experimentally identified models with models derived from first principles (such as FE models, also referred to as analytical models in this paper), which is especially useful when some parts are hard to model or when no model exists.

Dynamic substructuring has been mostly applied to FE models, while successful results of experimental substructuring are scarce in literature, see [16]. The most commonly used experimental substructuring techniques to date are the component mode synthesis (CMS) (see e.g. [16]) and frequency based substructuring (FBS) methods. The most common FBS method is the admittance coupling method proposed by Jetmundsen et al. [13]. Su and Juang [41] introduced a different approach using first-order statespace systems. A similar approach was also taken by 
Sjövall and Abrahamsson [39], later used and elaborated by Liljerehn, see [18].

The advantage of coupling state-space models manifests for experimental-analytical substructuring. Identifying a model of the assembled structure, e.g from data after performing FBS, is often more difficult as the dynamics of larger structures can be more complex. An easier approach to obtain a model of the assembled structure is to identify models from measured data of the substructures and apply state-space substructuring. One wide-spread method to identify a model from experimental data is subspace state-space system identification [20, 28, 42], e.g. N4SID [26]. Therefore, a substructuring technique for this model structure exploits the advantage of not having to transform the identified model to an appropriate model form, i.e. second order form for the CMS method.

It should be stressed that for the state-space synthesis method introduced in [39] to succeed the identified statespace systems must fulfil some physical constraints [39]. Therefore, due to noise and other imperfections in the experimental data the system identification procedure must be constrained in most situations, which is usually performed in a post-processing procedure $[17,40]$. Particularly, nonpassive state-space systems or systems that do not fulfil Newton's second law may cause unphysical coupling results [19].

The synthesis of substructures in experimental or experimental-analytical dynamic substructuring is problematic since in general not all degrees of freedom (DOFs) at the coupling interface can be measured as would be necessary to enforce strict compatibility. For some structures the connection points might not be accessible at all. This results in approximate coupling conditions, which can sometimes be very crude. The experimentally obtained data as well as the identified models must be of very high quality if successful synthesis is to be achieved. Furthermore, in experimental-analytical substructuring the experimentally identified structures mode shape basis can be inadequate for coupling, as generally experimental modes are obtained with free-free boundary conditions. To overcome these drawbacks, the transmission simulator was introduced for CMS by Allen et al. [4] and for FBS by Mayes et al. [25]. The method works by attaching a well-modelled additional structure, the transmission simulator, to the interface region of the structure of interest. The coupled system is then measured and an experimental model is identified from the acquired data. Thus, the interface region is mass-loaded and excited such that a more suitable mode shape basis can be obtained. This is because mass-loading the interfaces causes the interface region to be excited in a lower frequency region which (ideally) closely resembles the coupling conditions for the true coupling. Hence, the difficulty of measuring the rotations and forces at all connection points is avoided [24], which is generally necessary for coupling. The transmission simulator furthermore allows for joint properties to be included in the model if the joints of the transmission simulator resemble the joints of the system to be coupled. It is therefore advised to use a part of the actual assembled system as a transmission simulator if possible [4]. A model of the system of interest is obtained using an analytical model of the transmission simulator and removing its effect from the measured system [4]. To this end, dynamic substructuring is used, but here the measurement points on the transmission simulator are coupled to exactly the same points of the negative, analytical transmission simulator model. Thus, measuring the actual connection points is avoided. The compatibility conditions are fulfilled in a least-squares sense by the use of the modal constraints for fixture and subsystem (MCFS) method suggested by Allen et al. [4], and hence, MCFS is further also capable to compensate for measurement errors. An alternative constraint formulation is the connection point (CPT) method. CPT is not considered in this paper but has been applied to state-space substructuring in [34].

One hindrance of the transmission simulator is the occurrence of indefinite mass or stiffness matrices after decoupling, which yields non-physical results [3]. Techniques to overcome this problem were suggested by Mayes et al. [23], namely the modal scale factor method and added mass method. A new extension of the transmission simulator approach is the Craig-Bampton transmission simulator introduced by Kammer et al. [15], where fixed interface modes are used instead of free-free mode shapes. Alternative ideas to overcome the difficulties in experimental-analytical substructuring without the use of a transmission simulator have been proposed. One of them is the virtual coupling point and equivalent multi-point connection [36, 37] where translational measurements close to the actual connection point are used. D' Ambrogio and Fregolent suggested the extended and mixed interfaces for coupling [7]. These methods have not been considered in this paper.

The transmission simulator approach to dynamic substructuring has been used on the Society for Experimental Mechanics' (SEM) substructuring focus group's benchmark test-bed structure, the Ampair A600 wind turbine [22], in previous studies. In [31] Roettgen and Mayes coupled one blade to the hub three times to obtain a full rotor model using the hub as transmission simulator, whereas in [32] Rohe and Mayes coupled the rotor to the tower of the wind turbine, again using the hub as transmission simulator. The hub is a convenient transmission simulator since it is stiff compared to the blades and can be modelled fairly easily. Furthermore, the actual joints are used for connecting the interfaces yielding a realistic interface excitation.

In this paper, which is based on the work found in [9-11, $34,35]$, the transmission simulator approach is integrated 
into state-space substructuring to exploit their individual advantages listed above for experimental substructuring. The method is applied to state-space substructuring on the Ampair A600 test-bed. First, the MCFS transmission simulator method is translated into state-space. Thereafter the substructuring task of Roettgen and Mayes [31] is repeated. However, three different blades are measured in order to account for spread of dynamic properties found in the blades [11]. In Fig. 1 an overview of the coupling methodology is shown. First, the blades labelled 852, 828 and 790 are attached to the hub one at a time. These onebladed hub configurations are measured and experimental models identified using system identification. In assembling the models, the FE representation of the hub transmission simulator model is subtracted twice. Thus, the three-bladed hub dubbed "Assembly" (coupled) structure is obtained. For validation the same three blades are assembled to the hub simultaneously and measured. By comparing the frequency response functions (FRFs) and modal properties the quality of the assembled structure is evaluated. Furthermore, FRFs obtained with state-space substructuring, CMS as well as FE modelling will be compared.

The paper is structured as follows. In Section "Transmission Simulator in the State-Space Domain" the theory of the transmission simulator method in state-space is derived. In Section "Models" the experimental and finite element models are presented. The results of the substructuring are shown in Section "Substructuring Results" followed by a discussion of the results in Section "Discussion". The paper is concluded in Section "Conclusion". In Appendix A the classical state-space synthesis theory is presented to clarify some steps in the transmission simulator state-space method and in Appendix B the theory behind the MCFS in the modal domain from the paper of Allen et al. [4] is reiterated.

\section{Transmission Simulator in the State-Space Domain}

The equations of motion of a linear time-invariant system of some component $(s)$ can be written on first order form as

$$
\begin{aligned}
\dot{\boldsymbol{x}}^{(s)}(t) & =\boldsymbol{A}^{(s)} \boldsymbol{x}^{(s)}(t)+\boldsymbol{B}^{(s)} \boldsymbol{u}^{(s)}(t) \\
\boldsymbol{y}^{(s)}(t) & =\boldsymbol{C}^{(s)} \boldsymbol{x}^{(s)}(t)
\end{aligned}
$$

where $\boldsymbol{A}^{(s)} \in \mathbb{R}^{n^{(s)} \times n^{(s)}}, \boldsymbol{B}^{(s)} \in \mathbb{R}^{n^{(s)} \times n_{\mathrm{u}}^{(s)}}$ and $\boldsymbol{C}^{(s)} \in$ $\mathbb{R}^{n_{y}^{(s)} \times n^{(s)}}$ represent the state, input and displacement output matrices, respectively. Explicit time dependence will be dropped for brevity from here on. Here $\boldsymbol{x}^{(s)} \in \mathbb{R}^{n^{(s)} \times 1}$ is the state vector, $\boldsymbol{u}^{(s)} \in \mathbb{R}^{n_{\mathrm{u}}^{(s)} \times 1}$ the system input vector and $\boldsymbol{y}^{(s)} \in \mathbb{R}^{n_{y}^{(s)} \times 1}$ the system output vector. Note that for both displacement and velocity outputs, the relation for the feedthrough matrix $\boldsymbol{D}^{(s)}=\mathbf{0}$ holds since forces have a direct influence on acceleration only, according to Newton's second law.

For a mechanical system modelled with $\mathrm{FE}$, and with $\boldsymbol{q}^{(s)} \in \mathbb{R}^{m^{(s)} \times 1}$ the physical DOF vector, the second order differential equation can be rewritten to first order form with the state vector

$\boldsymbol{x}^{(s)}=\left[\begin{array}{c}\boldsymbol{q}^{(s)} \\ \dot{\boldsymbol{q}}^{(s)}\end{array}\right]$.

It follows that $n^{(s)}=2 m^{(s)}$. Then the state, input and output matrices are

$$
\begin{aligned}
& \boldsymbol{A}^{(s)} \triangleq\left[\begin{array}{cc}
\mathbf{0}^{(s)} & \boldsymbol{I}^{(s)} \\
-\boldsymbol{M}^{-1^{(s)}} \boldsymbol{K}^{(s)} & -\boldsymbol{M}^{-1^{(s)}} \boldsymbol{V}^{(s)}
\end{array}\right] \\
& \boldsymbol{B}^{(s)} \triangleq\left[\begin{array}{c}
\mathbf{0}^{(s)} \\
\boldsymbol{M}^{-1^{(s)}} \boldsymbol{P}_{\mathrm{u}}^{(s)}
\end{array}\right] \\
& \boldsymbol{C}^{(s)} \triangleq\left[\begin{array}{ll}
\boldsymbol{P}_{\mathrm{y}}^{(s)} & \mathbf{0}^{(s)}
\end{array}\right]
\end{aligned}
$$

where $\boldsymbol{M}^{(s)} \in \mathbb{R}^{m^{(s)} \times m^{(s)}}, \boldsymbol{V}^{(s)} \in \mathbb{R}^{m^{(s)} \times m^{(s)}}$ and $\boldsymbol{K}^{(s)} \in \mathbb{R}^{m^{(s)} \times m^{(s)}}$ represent the mass, damping and stiffness matrices, respectively. Furthermore, $\boldsymbol{P}_{\mathrm{u}}^{(s)} \in \mathbb{R}^{m^{(s)} \times n_{\mathrm{u}}(s)}$ and $\boldsymbol{P}_{\mathrm{y}}^{(s)} \in \mathbb{R}^{n_{\mathrm{y}}^{(s)} \times m^{(s)}}$ indicate the input and output location matrices, respectively, and $\boldsymbol{I}^{(s)} \in \mathbb{R}^{m^{(s)} \times m^{(s)}}$ is the identity matrix.

Here the state-space synthesis method developed by Sjövall and Abrahamsson [39] will be used. The method is briefly restated in Appendix A. For reference the transmission simulator for CMS is outlined in Appendix B. In this section we will first show how to define a negative state-space system. Then the MCFS transmission simulator technique will be developed for the state-space synthesis. In the subsequent, removing one structure from another will be denoted subtraction or decoupling of a system.

Fig. 1 Three one-bladed hubs are measured and coupled with two negative FE transmission simulators (TS)

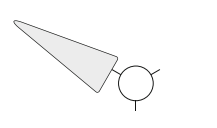

Blade 852 (measured)
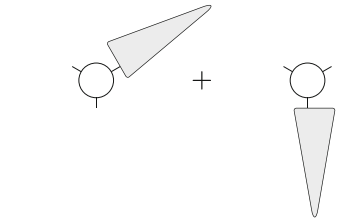

Blade 828 (measured)

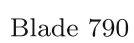

(measured)
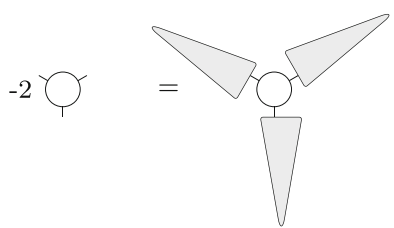

TS

$(\mathrm{FE})$
Assembly (coupled) 


\section{Negative State-Space System}

To substract a known substructure with the given realisation as in equation (3) from a known system it is possible to use existing substructuring methods. A negative mechanical system can be introduced through the negative mass $-\boldsymbol{M}^{(s)}$, damping $-\boldsymbol{V}^{(s)}$ and stiffness $-\boldsymbol{K}^{(s)}$ matrices, as shown in [4]. A negative state-space system of the same form as in equation (3) is then simply obtained as

$$
\begin{aligned}
\boldsymbol{A}^{(s)} & =\left[\begin{array}{cc}
\mathbf{0}^{(s)} & \boldsymbol{I}^{(s)} \\
-\left(-\boldsymbol{M}^{(s)}\right)^{-1} & \left(-\boldsymbol{K}^{(s)^{-1}}\right)-\left(-\boldsymbol{M}^{(s)}\right)^{-1}\left(-\boldsymbol{V}^{(s)^{-1}}\right)
\end{array}\right] \\
& =\left[\begin{array}{cc}
\mathbf{0}^{(s)} & \boldsymbol{I}^{(s)} \\
-\boldsymbol{M}^{(s)^{-1}} \boldsymbol{K}^{(s)^{-1}} & -\boldsymbol{M}^{(s)^{-1}} \boldsymbol{V}^{(s)^{-1}}
\end{array}\right] \\
\boldsymbol{B}^{(s)} & =\left[\begin{array}{c}
\mathbf{0}^{(s)} \\
-\boldsymbol{M}^{(s)^{-1}} \boldsymbol{P}_{u}^{(s)}
\end{array}\right]=-\left[\begin{array}{c}
\mathbf{0}^{(s)} \\
\boldsymbol{M}^{(s)^{-1}} \boldsymbol{P}_{\mathrm{u}}^{(s)}
\end{array}\right] \\
\boldsymbol{C}^{(s)} & =\left[\begin{array}{ll}
\boldsymbol{P}_{\mathrm{y}}^{(s)} & \mathbf{0}^{(s)}
\end{array}\right] .
\end{aligned}
$$

It can be seen that only the input matrix $\boldsymbol{B}^{(s)}$ is affected, i.e. need to be made negative to make a system negative.

\section{Modal Constraints for Fixture and Subsystem in State-Space Domain}

In the MCFS method the coupling is enforced by constraining the displacement of all points on the transmission simulator of the total system to be equal to the motion of the analytical transmission simulator model. The motion of the transmission simulator of the total system is described by $\boldsymbol{y}_{\mathrm{a}}^{\text {(tot) }} \in \mathbb{R}^{n_{\mathrm{a}} \times 1}$. The subscript a denotes the points on the transmission simulator that have been measured. By transforming the analytical, i.e. FE model, of the transmission simulator to state-space form, one can identify the motion of the same points, $\boldsymbol{y}_{\mathrm{a}}^{\text {(ts) }} \in \mathbb{R}^{n_{\mathrm{a}} \times 1}$.

Direct coupling of the measurement points on the transmission simulator is described by

$\overline{\boldsymbol{u}}_{\mathrm{a}}=\boldsymbol{u}_{\mathrm{a}}^{(\mathrm{tot})}+\boldsymbol{u}_{\mathrm{a}}^{(\mathrm{ts})} \quad$ and $\quad \overline{\boldsymbol{y}}_{\mathrm{a}}=\boldsymbol{y}_{\mathrm{a}}^{(\mathrm{tot})}=\boldsymbol{y}_{\mathrm{a}}^{(\mathrm{ts})}$.

With MCFS, these constraints are fulfilled in a least-square sense using the transmission simulator modal matrix $\boldsymbol{\Phi}_{\mathrm{a}}^{(\mathrm{ts})} \in$ $\mathbb{R}^{n_{\mathrm{a}} \times m_{\mathrm{ts}}}$, with $m_{\mathrm{ts}}$ the number of modes selected from the transmission simulator model,

$\overline{\boldsymbol{u}}_{\mathrm{mod}}=\boldsymbol{\Phi}_{\mathrm{a}}^{(\mathrm{ts})^{\mathrm{T}}} \boldsymbol{u}_{\mathrm{a}}^{(\mathrm{tot})}+\boldsymbol{\Phi}_{\mathrm{a}}^{(\mathrm{ts})^{\mathrm{T}}} \boldsymbol{u}_{\mathrm{a}}^{(\mathrm{ts})} \triangleq \boldsymbol{u}_{\bmod }^{(\mathrm{tot})}+\boldsymbol{u}_{\bmod }^{(\mathrm{ts})}$

$\overline{\boldsymbol{y}}_{\text {mod }}=\boldsymbol{y}_{\text {mod }}^{(\mathrm{tot})}=\boldsymbol{y}_{\text {mod }}^{(\mathrm{ts})}$

$\boldsymbol{y}_{\bmod }^{(\mathrm{tot})} \triangleq \boldsymbol{\Phi}_{\mathrm{a}}^{(\mathrm{ts})^{+}} \boldsymbol{y}_{\mathrm{a}}^{(\mathrm{tot})}$

$\boldsymbol{y}_{\mathrm{mod}}^{(\mathrm{ts})} \triangleq \boldsymbol{\Phi}_{\mathrm{a}}^{(\mathrm{ts})^{+}} \boldsymbol{y}_{\mathrm{a}}^{(\mathrm{ts})}$.

Here ${ }^{+}$denotes the Moore-Penrose pseudoinverse, and the subscript mod indicates modal coordinates. It is assumed that an FE model of the transmission simulator is available and therefore the whole modal matrix $\boldsymbol{\Phi}^{(\mathrm{ts})}$ is easily obtained and $\boldsymbol{\Phi}_{\mathrm{a}}^{(\mathrm{ts})}$ is the subset of the modal matrix associated with the measurement points on the transmission simulator. Note that $\boldsymbol{\Phi}_{\mathrm{a}}^{\text {(ts) }}$ must be full column rank.

The measured input and output vectors for the total and transmission simulator systems, with $(s)$ either (tot) or (ts), are partitioned as

$\boldsymbol{u}^{(s)}=\left[\begin{array}{l}\boldsymbol{u}_{\mathrm{a}}^{(s)} \\ \boldsymbol{u}_{\mathrm{b}}^{(s)}\end{array}\right] \quad$ and $\quad \boldsymbol{y}^{(s)}=\left[\begin{array}{l}\boldsymbol{y}_{\mathrm{a}}^{(s)} \\ \boldsymbol{y}_{\mathrm{b}}^{(s)}\end{array}\right]$.

Here the subscript $\mathrm{b}$ denotes the body coordinates not used in coupling. The partitioned input and output matrices are then

$$
\begin{aligned}
\boldsymbol{B}^{(s)} & =\left[\begin{array}{ll}
\boldsymbol{B}_{\mathrm{a}}^{(s)} & \boldsymbol{B}_{\mathrm{b}}^{(s)}
\end{array}\right] \\
\boldsymbol{C}^{(s)} & =\left[\begin{array}{l}
\boldsymbol{C}_{\mathrm{a}}^{(s)} \\
\boldsymbol{C}_{\mathrm{b}}^{(s)}
\end{array}\right] .
\end{aligned}
$$

Note also that the subscript a in $\boldsymbol{C}_{\mathrm{a}}^{(s)}$ is here used specifically to represent displacement outputs at the transmission simulator DOFs a. Introducing the modal coordinates from equation (6), the system inputs and outputs in equation (7) are expressed as

$$
\begin{aligned}
\boldsymbol{u}^{(s)} & =\left[\begin{array}{cc}
\boldsymbol{\Phi}_{\mathrm{a}}^{\left(\mathrm{ts}^{+}\right.} & \mathbf{0}^{(s)} \\
\mathbf{0}^{(s)} & \boldsymbol{I}^{(s)}
\end{array}\right]^{\mathrm{T}}\left[\begin{array}{c}
\boldsymbol{u}_{\mathrm{mod}}^{(s)} \\
\boldsymbol{u}_{\mathrm{b}}^{(s)}
\end{array}\right] \triangleq \boldsymbol{R}^{(s) \mathrm{T}} \hat{\boldsymbol{u}}^{(s)} \\
\boldsymbol{y}^{(s)} & =\left[\begin{array}{cc}
\boldsymbol{\Phi}_{\mathrm{a}}^{(\mathrm{ts})^{+}} & \mathbf{0}^{(s)} \\
\mathbf{0}^{(s)} & \boldsymbol{I}^{(s)}
\end{array}\right]^{+}\left[\begin{array}{c}
\boldsymbol{y}_{\bmod }^{(s)} \\
\boldsymbol{y}_{\mathrm{b}}^{(s)}
\end{array}\right] \triangleq \boldsymbol{R}^{(s)} \hat{\boldsymbol{y}}^{(s)} .
\end{aligned}
$$

\section{Decoupling in the State-Space Domain}

With the transformed inputs and outputs, the model $(s)$ is

$$
\begin{aligned}
\dot{\boldsymbol{x}}^{(s)} & =\boldsymbol{A}^{(s)} \boldsymbol{x}^{(s)}+\boldsymbol{B}^{(s)} \boldsymbol{R}^{(s)^{\mathrm{T}}} \hat{\boldsymbol{u}}^{(s)} \\
& \triangleq \boldsymbol{A}^{(s)} \boldsymbol{x}^{(s)}+\hat{\boldsymbol{B}}^{(s)} \hat{\boldsymbol{u}}^{(s)} \\
\hat{\boldsymbol{y}}^{(s)} & =\boldsymbol{R}^{(s)} \boldsymbol{C}^{(s)} \boldsymbol{x}^{(s)} \\
& \triangleq \hat{\boldsymbol{C}} \boldsymbol{x}^{(s)} .
\end{aligned}
$$

Note that for the (ts) system $\hat{\boldsymbol{B}}^{\text {(ts) }}$ is negative, see Section "Negative State-Space System". The system in equation (10) is then transformed to coupling form using the transformation

$\tilde{\boldsymbol{x}}^{(s)}=\boldsymbol{T}^{(s)} \boldsymbol{x}(s)=\left[\begin{array}{c}\dot{\boldsymbol{y}}_{\bmod }^{(s)} \\ \boldsymbol{y}_{\bmod }^{(s)} \\ \boldsymbol{x}_{\mathrm{b}}^{(s)}\end{array}\right]$

where $\boldsymbol{T}^{(s)}$ is defined in Appendix A. Thereafter, by coupling the negative system of the transmission simulator model (ts) to the total system (tot) the influence of the 
transmission simulator is removed and the system of interest is obtained. The final state-space system can be written

$\dot{\overline{\boldsymbol{x}}}=\overline{\boldsymbol{A}} \overline{\boldsymbol{x}}+\overline{\boldsymbol{B}} \overline{\boldsymbol{u}}$

$\overline{\boldsymbol{y}}=\overline{\boldsymbol{C}} \overline{\boldsymbol{x}}$

with the coupled state, input and output matrices and vectors

$$
\begin{aligned}
& \overline{\boldsymbol{A}} \triangleq\left[\begin{array}{cccc}
\overline{\boldsymbol{A}}_{\mathrm{vv}} & \overline{\boldsymbol{A}}_{\mathrm{vd}} & \overline{\boldsymbol{A}}_{\mathrm{vb}}^{(\mathrm{tot})} & \overline{\boldsymbol{A}}_{\mathrm{vb}}^{(\mathrm{ts})} \\
\boldsymbol{I} & \mathbf{0} & \mathbf{0} & \mathbf{0} \\
\mathbf{0} & \boldsymbol{A}_{\mathrm{bd}}^{(\mathrm{tot})} & \boldsymbol{A}_{\mathrm{bb}}^{(\mathrm{tot})} & \mathbf{0} \\
\mathbf{0} & \boldsymbol{A}_{\mathrm{bd}}^{(\mathrm{ts})} & \mathbf{0} & \boldsymbol{A}_{\mathrm{bb}}^{(\mathrm{ts})}
\end{array}\right] \\
& \overline{\boldsymbol{B}} \triangleq\left[\begin{array}{ccc}
\overline{\boldsymbol{B}}_{\mathrm{vv}} & \overline{\boldsymbol{B}}_{\mathrm{vb}}^{(\mathrm{tot})} & \overline{\boldsymbol{B}}_{\mathrm{vb}}^{\mathrm{ts})} \\
\mathbf{0} & \mathbf{0} & \mathbf{0} \\
\mathbf{0} & \boldsymbol{B}_{\mathrm{bb}}^{(\mathrm{tot})} & \mathbf{0} \\
\mathbf{0} & \mathbf{0} & \boldsymbol{B}_{\mathrm{bb}}^{(\mathrm{ts})}
\end{array}\right] \\
& \overline{\boldsymbol{C}} \triangleq\left[\begin{array}{cccc}
\mathbf{0} & \boldsymbol{I} & \mathbf{0} & \mathbf{0} \\
\boldsymbol{C}_{\mathrm{bv}}^{(\mathrm{tot})} & \boldsymbol{C}_{\mathrm{bd}}^{(\mathrm{tot})} & \boldsymbol{C}_{\mathrm{bb}}^{(\mathrm{tot})} & \mathbf{0} \\
\boldsymbol{C}_{\mathrm{bv}}^{(\mathrm{ts})} & \boldsymbol{C}_{\mathrm{bd}}^{(\mathrm{ts})} & \mathbf{0} & \boldsymbol{C}_{\mathrm{bb}}^{(\mathrm{ts})}
\end{array}\right] \\
& \overline{\boldsymbol{x}} \triangleq\left[\dot{\bar{y}}_{\text {mod }}^{\mathrm{T}} \overline{\boldsymbol{y}}_{\text {mod }}^{\mathrm{T}} \boldsymbol{x}_{\mathrm{b}}^{(\mathrm{tot})^{\mathrm{T}}} \boldsymbol{x}_{\mathrm{b}}^{(\mathrm{ts})^{\mathrm{T}}}\right]^{\mathrm{T}} \\
& \overline{\boldsymbol{u}} \triangleq\left[\overline{\boldsymbol{u}}_{\mathrm{mod}}^{\mathrm{T}} \overline{\boldsymbol{u}}_{\mathrm{b}}^{(\mathrm{tot})^{\mathrm{T}}} \overline{\boldsymbol{u}}_{\mathrm{b}}^{(\mathrm{ts})^{\mathrm{T}}}\right]^{\mathrm{T}} \\
& \overline{\boldsymbol{y}} \triangleq\left[\overline{\boldsymbol{y}}_{\bmod }^{\mathrm{T}} \boldsymbol{y}_{\mathrm{b}}^{(\mathrm{tot})^{\mathrm{T}}} \boldsymbol{y}_{\mathrm{b}}^{(\mathrm{ts})^{\mathrm{T}}}\right]^{\mathrm{T}} \text {. }
\end{aligned}
$$

The matrix partitions above are obtained as

$$
\begin{aligned}
\boldsymbol{K} & =\left(\boldsymbol{B}_{\mathrm{vv}}^{(\mathrm{tot})}-\boldsymbol{B}_{\mathrm{vv}}^{(\mathrm{ts})}\right)^{-1} \\
\overline{\boldsymbol{A}}_{\mathrm{vv}} & =\boldsymbol{B}_{\mathrm{vv}}^{(\mathrm{tot})} \boldsymbol{K} \boldsymbol{A}_{\mathrm{vv}}^{(\mathrm{ts})}-\boldsymbol{B}_{\mathrm{vv}}^{(\mathrm{ts})} \boldsymbol{K} \boldsymbol{A}_{\mathrm{vv}}^{(\mathrm{tot})} \\
\overline{\boldsymbol{A}}_{\mathrm{vd}} & =\boldsymbol{B}_{\mathrm{vv}}^{(\mathrm{tot})} \boldsymbol{K} \boldsymbol{A}_{\mathrm{vd}}^{(\mathrm{ts})}-\boldsymbol{B}_{\mathrm{vv}}^{(\mathrm{ts})} \boldsymbol{K} \boldsymbol{A}_{\mathrm{vd}}^{(\mathrm{tot})} \\
\overline{\boldsymbol{A}}_{\mathrm{vb}}^{(\mathrm{tot})} & =-\boldsymbol{B}_{\mathrm{vv}}^{(\mathrm{ts})} \boldsymbol{K} \boldsymbol{A}_{\mathrm{vb}}^{(\mathrm{tot})} \\
\overline{\boldsymbol{A}}_{\mathrm{vb}}^{(\mathrm{ts})} & =\boldsymbol{B}_{\mathrm{vv}}^{(\mathrm{tot})} \boldsymbol{K} \boldsymbol{A}_{\mathrm{vb}}^{(\mathrm{ts})} \\
\overline{\boldsymbol{B}}_{\mathrm{vv}} & =-\boldsymbol{B}_{\mathrm{vv}}^{(\mathrm{tot})} \boldsymbol{K} \boldsymbol{B}_{\mathrm{vv}}^{(\mathrm{ts})} \\
\overline{\boldsymbol{B}}_{\mathrm{vb}}^{(\mathrm{tot})} & =-\boldsymbol{B}_{\mathrm{vv}}^{(\mathrm{ts})} \boldsymbol{K} \boldsymbol{B}_{\mathrm{vb}}^{(\mathrm{tot})} \\
\overline{\boldsymbol{B}}_{\mathrm{vb}}^{(\mathrm{ts})} & =-\boldsymbol{B}_{\mathrm{vv}}^{(\mathrm{tot})} \boldsymbol{K} \boldsymbol{B}_{\mathrm{vb}}^{(\mathrm{ts})} .
\end{aligned}
$$

The subscripts $\mathrm{d}$ and $\mathrm{v}$ denote displacement and velocity, respectively. This is further explained in more detail in Appendix A, where the original state-space synthesis algorithm from [39] is outlined. The non-coupling form input matrix $\hat{\boldsymbol{B}}^{\text {(ts) }}$ and hence also the partitions $\boldsymbol{B}_{\mathrm{vv}}^{\text {(ts) }}$ and $\boldsymbol{B}_{\mathrm{vb}}^{(\mathrm{ts})}$ are here considered positive and the minus sign is therefore explicitly shown for clarity.

In cases when several copies of one substructure are coupled, special care must be taken for the term $\overline{\boldsymbol{B}}_{\mathrm{vv}}$. In general, state-space synthesis of two systems (tot), (ts) can be shown to be equal to extracting the second-order differential equation of each model and adding them [38].
If the total system will be coupled to $k_{\mathrm{ts}}$ copies of the transmission simulator, this sum is

$$
\begin{gathered}
\boldsymbol{B}_{\mathrm{vv}}^{(\mathrm{tot})^{-1}} \ddot{y}_{\mathrm{c}}^{(\mathrm{tot})}+k_{\mathrm{ts}} \boldsymbol{B}_{\mathrm{vv}}^{(\mathrm{ts})^{-1}} \ddot{y}_{\mathrm{c}}^{(\mathrm{ts})} \\
=\boldsymbol{B}_{\mathrm{vv}}^{(\mathrm{tot})^{-1}}\left(\boldsymbol{A}_{\mathrm{vv}}^{(\mathrm{tot})} \dot{y}_{\mathrm{c}}^{(\mathrm{tot})}+\boldsymbol{A}_{\mathrm{vd}}^{(\mathrm{tot})} y_{\mathrm{c}}^{(\mathrm{tot})}+\boldsymbol{A}_{\mathrm{vb}}^{(\mathrm{tot})} y_{\mathrm{b}}^{(\mathrm{tot})}\right. \\
\left.+\boldsymbol{B}_{\mathrm{vv}}^{(\mathrm{tot})} u_{\mathrm{c}}^{(\mathrm{tot})}+\boldsymbol{B}_{\mathrm{vb}}^{(\mathrm{tot})} u_{\mathrm{b}}^{(\mathrm{tot})}\right) \\
+k_{\mathrm{ts}} \boldsymbol{B}_{\mathrm{vv}}^{(\mathrm{ts})^{-1}}\left(\boldsymbol{A}_{\mathrm{vv}}^{(\mathrm{ts})} \dot{y}_{\mathrm{c}}^{(\mathrm{ts})}+\boldsymbol{A}_{\mathrm{vd}}^{(\mathrm{ts})} y_{\mathrm{c}}^{(\mathrm{ts})}+\boldsymbol{A}_{\mathrm{vb}}^{(\mathrm{ts})} y_{\mathrm{b}}^{(\mathrm{ts})}\right. \\
\left.+\boldsymbol{B}_{\mathrm{vv}}^{(\mathrm{ts})} u_{\mathrm{c}}^{(\mathrm{ts})}+\boldsymbol{B}_{\mathrm{vb}}^{(\mathrm{ts})} u_{\mathrm{b}}^{(\mathrm{ts})}\right) .
\end{gathered}
$$

This is equal to dividing $\boldsymbol{B}_{\mathrm{vv}}^{(\mathrm{ts})}$ by $k_{\mathrm{ts}}$ in equation (14). Yet, as shown in equation (27) in Appendix B, several copies of one system are expressed by only multiplying the mass, stiffness, and damping parameters. The input matrix for the forces remains equal. Therefore, the coupling conditions for input and output (cf. Equation (19) in Appendix A) remain also unmodified. To account for this, the definition of $\overline{\boldsymbol{B}}_{\mathrm{vv}}$ must change if one system is to be coupled multiple times

$\overline{\boldsymbol{B}}_{\mathrm{vv}}=\frac{1+k_{\mathrm{ts}}}{k_{\mathrm{ts}}} \boldsymbol{B}_{\mathrm{vv}}^{(\mathrm{tot})} \boldsymbol{K} \boldsymbol{B}_{\mathrm{vv}}^{(\mathrm{ts})}$.

\section{Models}

In this section the FE models of the blade, bracket and hub of an Ampair A600 wind turbine are presented. Further, the models derived from vibration experiments are described along with the system identification procedure used to derive them.

\section{Finite Element Models}

In this paper three FE models are used for three different components; hub, bracket and blade. Siemens FEMAP v11.1.0 with NX Nastran has been used to model and solve the FE models, respectively. The brackets attached to the hub can be seen in Fig. 2. This is also the transmission simulator used in this paper, in accordance with [31]. Details on the FE models are given in Table 1. The density of the hub and bracket FE models are chosen to represent the mass of the weighted components to ensure that the total mass of the models corresponds to that measured, see Table 2. In cases with mounted bolts the density of the bracket FE model is increased to $\rho=5000 \mathrm{~kg} / \mathrm{m}^{3}$. The FE models of the hub and brackets are further described in Scheel [34] and Gibanica [9]. In Fig. 3 the FE model of the blade is shown attached to the transmission simulator. The outer composite layer of the blade was predominately modelled using rectangular, with a few triangular elements. The blade FE model was calibrated to a blade later subject to destructive material testing by Johansson et al. [14], 
Fig. 2 FE model of the hub and brackets (a) front view and (b) rear view. The hub and brackets system was also used as transmission simulator in this study. The marked positions indicate sensor positions as mounted in the experiments for triaxial sensors on positions 1 to 9 , marked with grey circles, and uniaxial sensors on positions 11 to 19 , marked with white circles

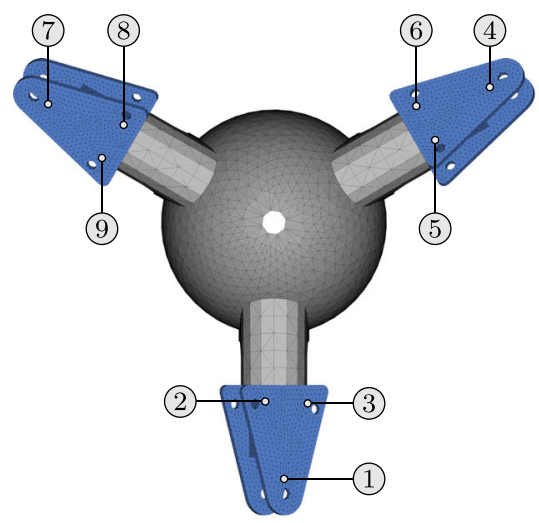

(a) Front view

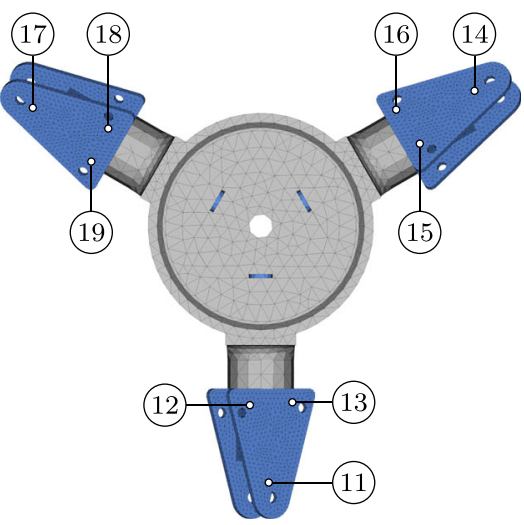

(b) Rear view which also describes the material model in detail. The blade model is not recalibrated towards any of the blades used in this paper. It should be noted that Gibanica et al. [11] showed that there exists a considerable spread between the blades. The interfaces between the bracket and blade, bracket and shaft, and shaft and hub are modelled as flexible connections using the CWELD element.

In Fig. 3 the FE model of the blade attached to the transmission simulator is shown. This model's rigid body modes are later used in the system identification step further described in Section "System Identification Methodology". In Fig. 4 the fully assembled system is shown, i.e. the assembly with hub, brackets and three blades.

\section{Experimental Set-up}

Four set-ups were measured. Each of the three blades was measured with the hub attached (acting as transmission simulator) to gather data for identification of the experimentally derived models to be coupled. Thereafter all three blades were connected to the hub to acquire a true or reference model to which the substructuring results are compared. The hub was filled with epoxy to remove auxiliary system dynamics from internal parts and the brackets were fixed to the hub, see Gibanica [9] for further details. In the onebladed hub measurements each blade was assembled to its specific bracket to replicate the full system configuration as close as possible. The blades were assembled to the brackets by bolts with a tightening torque of $21.69 \mathrm{Nm}(16 \mathrm{lbf}-\mathrm{ft})$ according to the SEM focus group's specifications [33].

The measurements were performed in a free-free boundary configuration with the structures hung in long, thin, high strength polyester lines. The lines were attached to a supporting steel structure. The rigid body modes in each experiment were thus well below the first flexible system mode. The experimental configurations are shown in Fig. 5.

Triaxial PCB Piezotronic type 356A03 accelerometers weighting $1 \mathrm{~g}$ and uniaxial PCB Piezotronic type PCB $352 \mathrm{C} 22 / \mathrm{NC}$ accelerometers weighting $0.5 \mathrm{~g}$ were used in all experiments. The fully assembled true system was measured with 9 triaxial and 24 uniaxial accelerometers while the three one-bladed hubs were measured with 10 triaxial and 25 uniaxial accelerometers. The total additional mass added to the systems was 21 and $22.5 \mathrm{~g}$, respectively, which was considered negligible. The accelerometer positions for the one-bladed hub are shown in Fig. 3 and for the fully assembled system in Fig. 4.

Mayes and Arviso [24] show that the conditioning of the transmission simulator mode shape matrix $\boldsymbol{\Phi}_{\mathrm{a}}^{(\mathrm{ts})}$ is greatly influenced by the accelerometer locations on the transmission simulator. Therefore, numerical models were used to devise the best placement from the available locations with respect to the mode shape matrix condition number. Three triaxial accelerometers were placed on the

Table 1 Details of the FE model. For each component the element and material type is given as well as the number of elements, Young's modulus $\mathrm{E}$ in $\mathrm{GPa}$ and density $\rho$ in $\mathrm{kg} / \mathrm{m}^{3}$

\begin{tabular}{|c|c|c|c|c|c|}
\hline Component & Element & \# elements & Material & $\mathrm{E}$ & $\rho$ \\
\hline Bracket, shaft with bolts & 10-noded solid parabolic CTETRA & 20126 & linear elastic isotropic & 200 & 5000 \\
\hline Bracket, shaft (without bolts) & 10-noded solid parabolic CTETRA & 20126 & linear elastic isotropic & 200 & 4050 \\
\hline Hub & 10-noded solid parabolic CTETRA & 11036 & linear elastic isotropic & 200 & 2095 \\
\hline Blade core & 4-noded solid linear CTETRA & 82577 & linear elastic isotropic & 1.67 & 818 \\
\hline Blade outer layer & rectangular and triangular shell CQUAD4 and CTRIA & 13320 & linear elastic orthotropic & 1.67 & 818 \\
\hline
\end{tabular}


Table 2 Measured masses, masses of the FE model and mass error $(\%)$. The measurements were performed using a scale with a precision of $0.1 \mathrm{~g}$ and the densities in the FE model calculated such that they resemble the measured weights

\begin{tabular}{llll}
\hline Component & Mass in kg & FE mass in kg & error \\
\hline Hub [9, 34] & 2.365 & 2.413 & 2.0 \\
Bracket, shaft, averaged [9] & 0.447 & 0.422 & -5.7 \\
Bracket, shaft, bolts [9] & 0.513 & 0.521 & 1.6 \\
\hline
\end{tabular}

front side of the bracket near the bolts, and three uniaxial accelerometers at the same locations on the opposite side of the bracket, as seen in Fig. 2. The accelerometer locations on the blade for the one-bladed hub were chosen among the locations used by Harvie and Avitabile [12], while the accelerometer locations on the fully assembled system were selected such that symmetry was preserved. Further details regarding the accelerometer location selection can be found in Scheel [34].

Shaker excitation was used as input for the experimental models used in this paper. The shaker was a The Modal Shop Inc., type K2007E01 with a nylon stinger approximately $85 \mathrm{~mm}$ in length. The excitation force was measured with a Brüel \& Kjær force sensor type 8203 weighting $3.2 \mathrm{~g}$ with an IEPE converter 2647B attached to the components through a stinger attachment plate that was glued to the test object. Impact testing was used to find the best input locations and assess the system's reciprocity. For the fully assembled system, locations 3, 6 and 9 were found to the give best results, while for the one-bladed hub locations 1 , 4 and 7 for systems 790,828 and 852 , respectively, were found to produce the best results. Periodic chirp excitation was used with different amplitude levels to determine the linear range of the system. The data used in system identification was gathered with a stepped multisine excitation using AbraDAQ [1] to minimise noise and hence obtain good estimates of the antiresonances. Further details regarding the measurement campaign can be found in Scheel [34].

\section{System Identification Methodology}

The state-space substructuring method relies on identified first order models from experimental data. Here, system identification is used to this end, see Ljung [19]. Specifically, in this paper a subspace state-space method has been used, implemented in the MATLAB System Identification Toolbox under the name N4SID and described in literature by McKelvey et al. [26].

The identification procedure requires only the number of system states to be provided by the user. This however requires that great care is taken in understanding the experimental system at hand. FE models can be used to guess an initial system order, e.g. [17]. It must be stressed that the correct model order is decisive for a successful substructuring procedure. Furthermore, because only one input location is used for each one-bladed hub the other connection points' input must be estimated as coupling requires the input output relation be known for all connection DOFs. From the identified state-space model the input matrix $\boldsymbol{B}$ can be populated for the missing inputs using modal data under the assumption of reciprocity, through Maxwell-Betti's reciprocal theorem $[8,21,29]$.

A drawback with the system identification procedure (for substructuring that requires physical systems) is that it does not necessarily estimate physically consistent models. Stability can easily be enforced using MATLAB's System Identification Toolbox, but passivity and Newton's second law can be, and many times are, violated, in that implementation. Synthesis of two non-passive state-space systems can yield an unstable synthesised system [39], shown in Gibanica [9]. A passive system is a system with non-negative energy dissipation, indicating a non-negative real part of the drive point FRF for linear reciprocal systems, see Sjövall and Abrahamsson [39]. This implies a phase between $\left[-90^{\circ}, 90^{\circ}\right]$ for mobility data. Recently, an approach to enforce passivity has been proposed by Liljerehn and Abrahamsson [19] using the fact that the receptance condition ensures the response at the drive point to be in the direction of the excitation force. This
Fig. 3 FE model of the one-bladed hub. The marked positions indicate sensors as mounted in the experiments for triaxial sensors on positions 1 to 10 , marked with grey circles, and uniaxial sensors on positions 11 to 35 , marked with white circles, of which 11 to 19 are located on the back side of the brackets, see Fig. 2

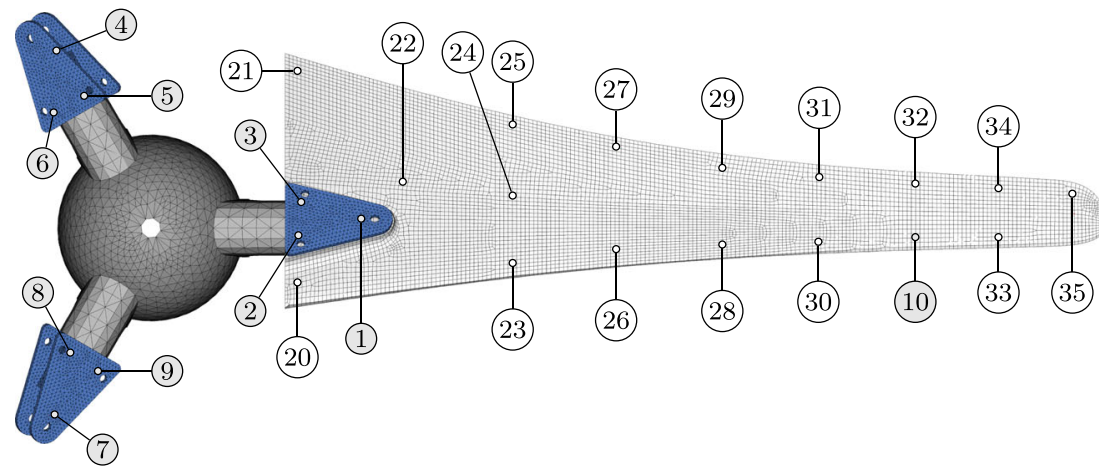


Fig. 4 FE model of the fully assembled system. The marked positions indicate sensors as mounted in the experiments for triaxial sensors on positions 1 to 9, marked with grey circles, uniaxial sensors on positions 11 to 19 which are on the back side of the brackets, see Fig. 2, and uniaxial sensors on positions 20 to 34 , marked with white circles

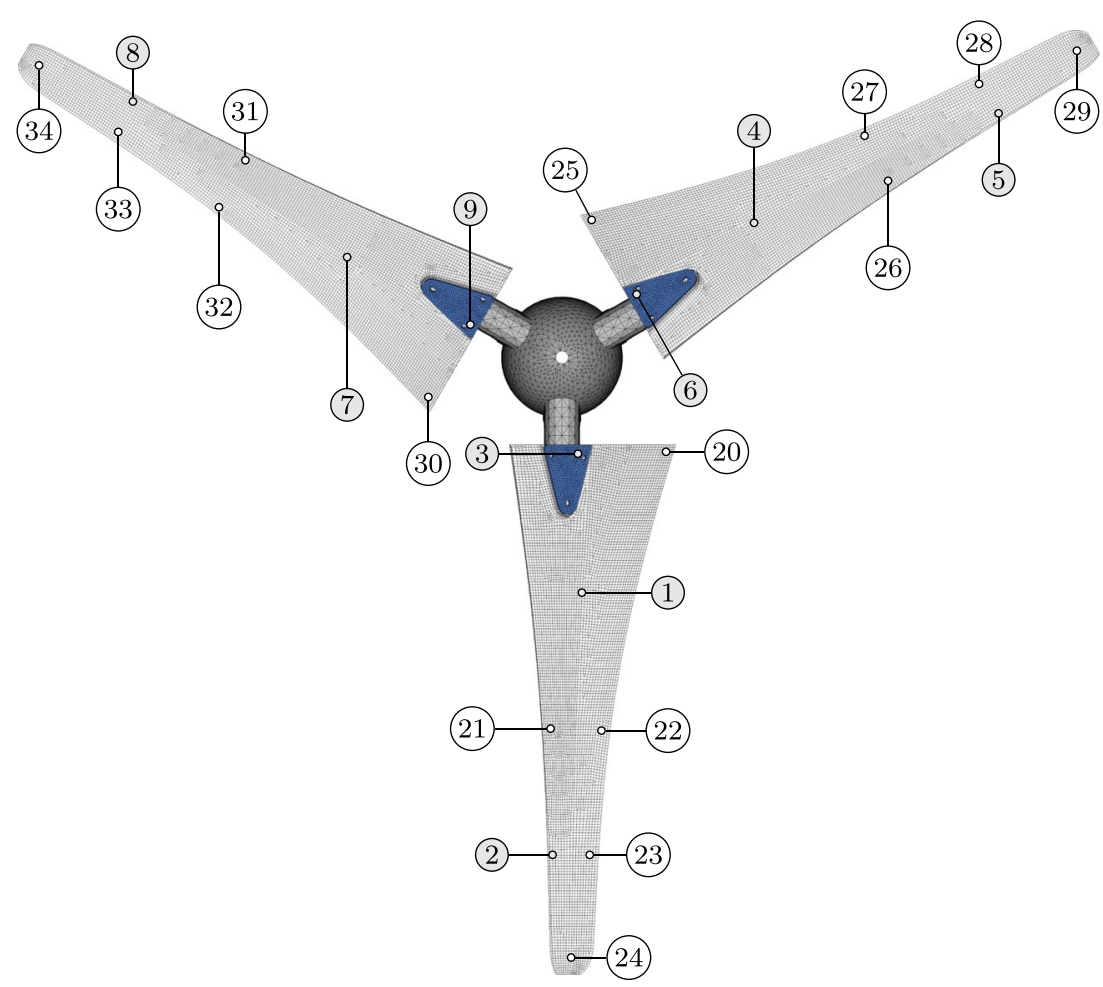

method is used in this paper. To ensure full physical consistency of the identified models from experimental data it is necessary to verify that Newton's second law holds, which need not be true due to measurement and truncation errors. From Newton's second law it is known that the force is proportional to acceleration. No such relation exists between force and velocity or displacement. Therefore, $\boldsymbol{D}_{\mathrm{d}}$ and $\boldsymbol{D}_{\mathrm{v}}=\boldsymbol{C}_{\mathrm{d}} \boldsymbol{B}=\mathbf{0}$ must hold, where subscripts d and $\mathrm{v}$ denote displacements and velocities, respectively.

The three one-bladed hubs were identified in an identical manner. First the influence from rigid body modes was removed using an FE model of the one-bladed hub, seen in Fig. 3. Then a model was identified from the stepped multisine data with 7 modes in the frequency range 10 to $400 \mathrm{~Hz}$ for accelerance data. An additional mode was included, located outside the $400 \mathrm{~Hz}$ range, to account for high frequency residuals. Accelerometers 24, 27 and 31 for structure 790 and accelerometers 24, 26, 28 and 31 for structure 828 and 852 were discarded from the identified models due to unresolved deficiencies in the measurement signals. Omitting the data from these sensors improved the quality of the identified model. Reciprocity and passivity
Fig. 5 Experimental set-up showing a the one-bladed hub assembly (figure from [35]) and b the fully assembled system

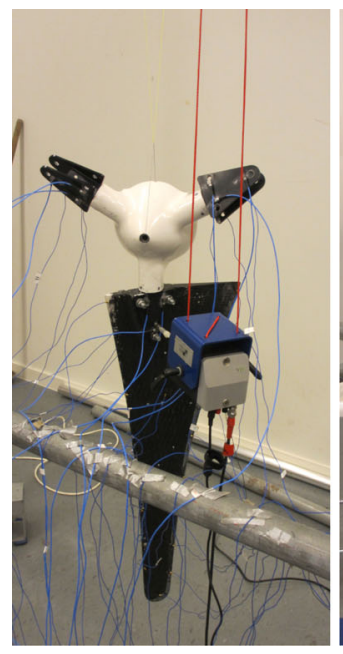

(b) One-bladed hub

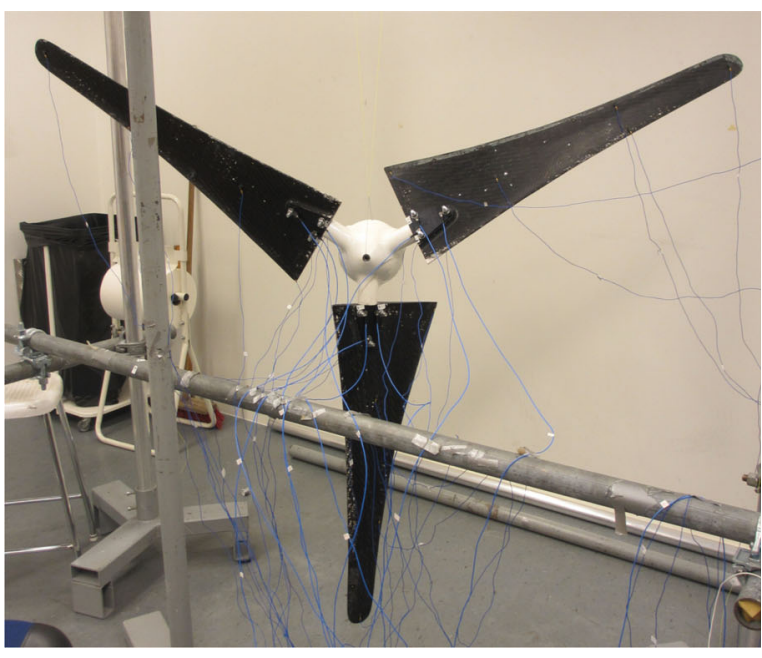

(a) Full system 
Table 3 Identified modal parameters, frequency $f(\mathrm{~Hz})$ and modal damping ratio $\xi$ $(\%)$, for the first seven modes of the one-bladed hubs used in substructuring compared to the parameters of the FE model. Further, the frequency errors $\Delta f(\%)$ between the identified models and the FE model are shown

\begin{tabular}{|c|c|c|c|c|c|c|c|c|c|c|}
\hline \multirow[b]{2}{*}{ \# } & \multirow{2}{*}{$\begin{array}{l}\text { FE } \\
f\end{array}$} & \multicolumn{3}{|c|}{ One-bladed hub 790} & \multicolumn{3}{|c|}{ One-bladed hub 828} & \multicolumn{3}{|c|}{ One-bladed hub 852} \\
\hline & & $f$ & $\Delta f$ & $\xi$ & $f$ & $\Delta f$ & $\xi$ & $f$ & $\Delta f$ & $\xi$ \\
\hline 1 & 30.32 & 33.11 & 9.2 & 1.34 & 31.38 & 3.6 & 1.33 & 31.51 & 4.0 & 1.50 \\
\hline 2 & 89.72 & 87.57 & -2.4 & 1.24 & 89.75 & 0.3 & 1.32 & 90.42 & 1.0 & 1.22 \\
\hline 3 & 180.90 & 165.27 & -8.6 & 1.71 & 164.89 & -8.0 & 1.69 & 165.40 & -7.8 & 1.59 \\
\hline 4 & 191.46 & 190.72 & -0.4 & 2.30 & 176.01 & -8.0 & 4.84 & 178.87 & -6.4 & 3.24 \\
\hline 5 & 234.06 & 208.83 & -10.8 & 1.68 & 199.36 & -11.2 & 1.52 & 201.68 & -10.2 & 1.48 \\
\hline 6 & 330.78 & 300.84 & -9.1 & 1.79 & 301.15 & -8.1 & 2.35 & 302.01 & -7.8 & 2.21 \\
\hline 7 & 341.88 & 316.97 & -7.3 & 1.89 & 318.16 & -6.4 & 1.77 & 314.53 & -7.4 & 1.48 \\
\hline
\end{tabular}

was then enforced on mobility data. Newton's second law was verified on receptance data where it was found that $\boldsymbol{C}_{\mathrm{d}} \boldsymbol{B}$ was of order $10^{-4} \mathrm{~m} / \mathrm{N}$ which was considered good enough for the application at hand. Finally, rigid body modes from the FE model of the one-bladed hub were included in the identified state-space models. The modal damping ratios for all rigid body modes were set to $\xi=0.1 \%$ to prevent numerical issues.

\section{Experimental Models}

In Table 3 the resonance frequency $f$ is shown for the first seven modes of the three one-bladed hub structures along with the modal damping ratio $\xi$ and relative frequency error $\Delta f$ between the FE model and the identified models. It can be seen that the identified models differ considerably, and that their difference to the FE model is also considerable. The largest deviation is found for flexible mode five. It should be repeated that the FE model of the blade was calibrated towards another blade and that it is known that the blades show a considerable spread in modal properties [11].

A representative FRF comparison from the system identification of one-bladed hub 852 is presented in Fig. 6(a). It can be seen that the identified model fit is good. The other two one-bladed hubs produced similar results and are not shown here, but can be found in Scheel [34]. To the right of the same figure a modal assurance criterion (MAC) [2] comparison between the identified model and the FE model of the blade and hub system is shown. It can be seen that the MAC value of the first four modes is above 0.9 which is considered good. The fifth mode is a combination of a bending and an in-plane motion mode resulting in a lower MAC value. From Table 3 it was also noted that this mode's resonance frequency deviated the most from the FE model. Mode six and seven are fairly close in frequency and show a cross correlation. For the two other blades these modes were identified as a linear combination of the FE modes. The last mode is not physical, but was added to account for a high frequency residual, as mentioned above.

A model of the fully assembled system was identified for use as reference or true model. The identified model can be seen in Fig. 7, and is seen to correspond well to the experimental data. The MAC values are also shown between the identified and the corresponding FE model. It can be seen that they are fairly low for many modes. This can be explained by the symmetry of the structure. Due to the symmetry, the flexible modes can be sorted in groups of three with at least two of them close in frequency (see Table 4). If modes are close in frequency, the calculated eigenvectors of the identified system span the subspace associated with these modes, they can be arbitrarily rotated.
Fig. 6 FRF from the measurement and the identified model of the one-bladed hub 852 (a). Input at location 7 and output at location 10 , both in out-of-plane direction as visualized in the figure. The MAC plot (b) compares the identified flexible mode shapes with the FE mode shapes

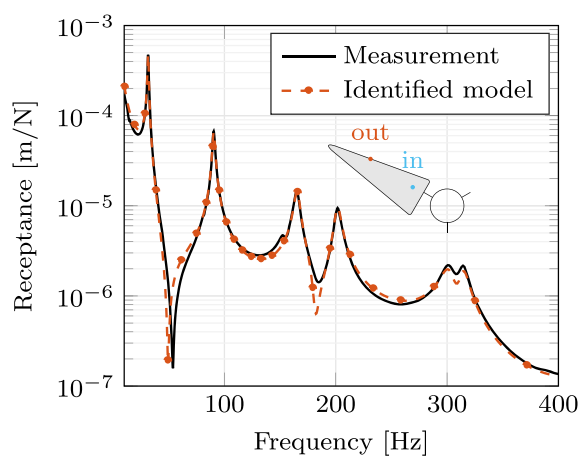

(a)

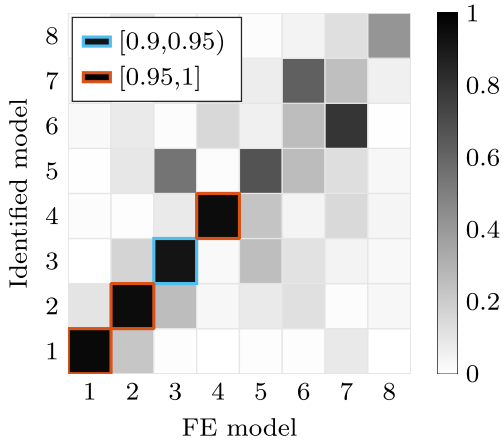

(b) 
Fig. 7 FRF from the measurements and the identified model of the three-bladed hub (a). Input at location 3 in out-of-plane direction and output location 28 as visualized in the figure. The MAC plot (b) compares the identified flexible mode shapes with the FE mode shapes. The coloured frames are connected to the angles between the subspaces spanned by mode shape groups

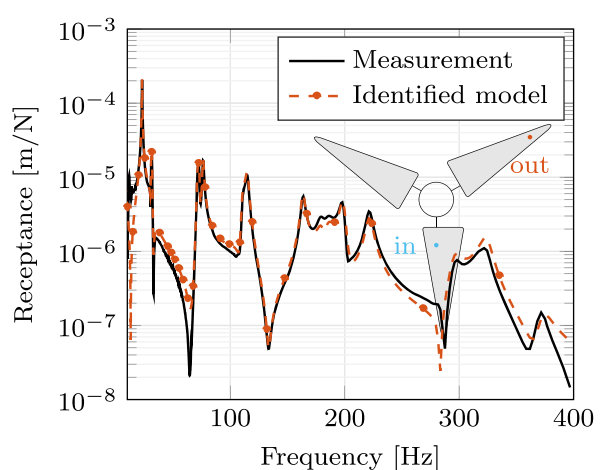

(a)

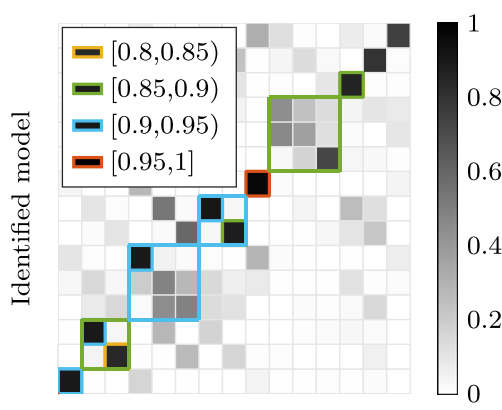

FE model

(b)
Thus, the identified mode shapes are approximately (close to) a linear combination of the associated FE mode shape vectors yielding low MAC values. To account for that, the angle between the subspaces spanned by the identified eigenvectors and the FE mode shapes is calculated for modes close in frequency [6]. If the angle is close to zero, the subspaces align well, and the corresponding mode shapes replicate the same motion. In the subsequent MAC plots, the squared cosine of the angle between the subspaces is illustrated by a coloured frame. Note that the squared cosine of the angle between subspaces spanned by only one vector each is equal to the MAC value. Applying this metric, it can be seen that the first 9 flexible modes correlate well with the FE model. The identified parameters can be found in Table 4. Note also that the FE modes in the table are sorted, taking into account mode mix-up. Curiously, the first FE resonance frequency match the measured resonance perfectly even though the first resonance frequency of all measured one-bladed hubs is higher than in their corresponding FE model.

\section{Substructuring Results}

The presented method is applied to the Ampair A600 wind turbine. It is of interest to know whether an experimentalanalytical modelling of the blade and hub system yields better results compared to a complete FE model of the same system where large uncertainties are associated with the blade FE models.

The resulting FRF from the substructuring procedure for one output channel can be seen in Fig. 8. The chosen channel is representative for the substructuring results. The experimentally identified model of the full assembly is
Table 4 Modal parameters, frequency $f(\mathrm{~Hz})$ and modal damping ratio $\xi(\%)$, of the truth and the coupled systems (substr) for the first five flexible mode groups for the three-bladed hub compared to the FE model. The resonance frequency and modal damping errors $\Delta f(\%)$ and $\Delta \xi(\%)$, respectively, between the systems are also shown. The FE modes are sorted to obtain the highest MAC correlation with the identified modes

\begin{tabular}{|c|c|c|c|c|c|c|c|}
\hline \multirow{2}{*}{$\begin{array}{l}\text { FE } \\
f\end{array}$} & \multicolumn{2}{|l|}{ true } & \multicolumn{2}{|l|}{ substr } & \multicolumn{2}{|l|}{$\Delta f$} & \multirow{2}{*}{$\begin{array}{l}\Delta \xi \\
\text { true-substr }\end{array}$} \\
\hline & $f$ & $\xi$ & $f$ & $\xi$ & FE-true & true-substr & \\
\hline 22.91 & 22.91 & 0.80 & 24.57 & 1.05 & 0.0 & 7.24 & 31.02 \\
\hline 30.34 & 30.69 & 0.85 & 30.96 & 1.37 & 1.2 & 0.89 & 62.21 \\
\hline 30.34 & 31.57 & 0.89 & 31.94 & 1.35 & 4.1 & 1.17 & 50.78 \\
\hline 83.01 & 71.34 & 1.23 & 75.62 & 1.05 & -14.1 & 6.01 & -14.36 \\
\hline 83.02 & 72.71 & 1.10 & 78.04 & 1.14 & -12.4 & 7.32 & 3.50 \\
\hline 79.12 & 76.19 & 0.86 & 82.40 & 1.09 & -3.7 & 8.15 & 26.70 \\
\hline 134.52 & 110.62 & 1.39 & 118.57 & 1.43 & -17.8 & 7.18 & 2.90 \\
\hline 134.49 & 114.93 & 1.27 & 120.44 & 1.29 & -14.5 & 4.80 & 1.32 \\
\hline 176.45 & 163.46 & 1.03 & 168.23 & 1.52 & -7.4 & 2.92 & 47.30 \\
\hline 189.63 & 179.78 & 1.90 & 174.01 & 4.26 & -5.2 & -3.21 & 124.34 \\
\hline 188.85 & 181.36 & 1.89 & 176.18 & 2.93 & -4.0 & -2.86 & 55.17 \\
\hline 189.63 & 185.50 & 2.43 & 185.33 & 2.00 & -2.2 & -0.09 & -17.59 \\
\hline 205.45 & 198.12 & 1.24 & 195.41 & 1.80 & -3.6 & -1.36 & 44.93 \\
\hline 205.45 & 200.24 & 1.42 & 199.40 & 1.75 & -2.5 & -0.42 & 23.11 \\
\hline 246.91 & 221.58 & 1.37 & 212.91 & 2.33 & -10.3 & -3.91 & 69.69 \\
\hline
\end{tabular}


Fig. 8 FRF of the three-bladed hub. Input/output location at 34 (cf. Fig. 4) as visualized in the sketch

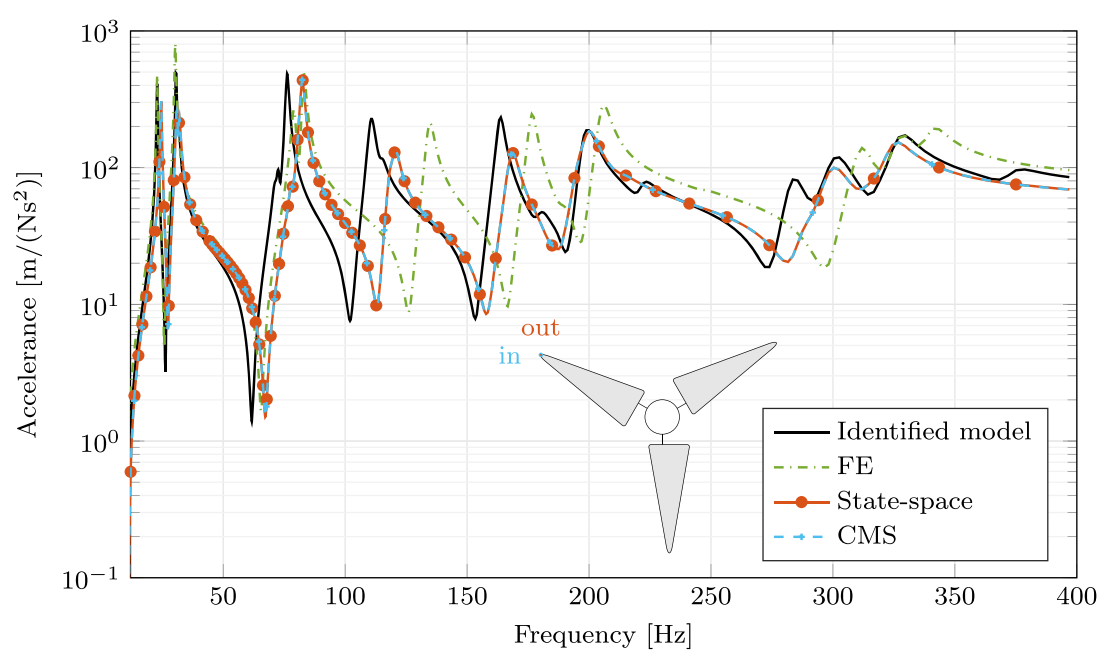

the reference, denoted true model. To compute the FRF of the FE model, the modal damping ratios from the true model are mapped in the FE model in this figure. Note that substructuring results are shown both for state-space coupling and CMS and that both methods yield the same results. In Fig. 9 the MAC values are shown between the true system and the substructured model. According to the angle between the subspaces in the MAC plot, the first nine flexible modes are well captured. In Table 4 the resonance frequencies and damping values are shown as well as the associated errors. It can be seen that the overall behaviour of the system is captured well. The first nine resonance frequencies of the substructured system are higher than for the the true system, and the damping is clearly overestimated (up to $124 \%$ for mode 10). For the last six modes the resonance frequencies of the substructured system are lower compared to the true system. In general however, the substructured model represents the true system
Fig. 9 The MAC plot shows a comparison between the first flexible mode shapes of the true system and the state-space substructuring results. The coloured frames are connected to the angles between the subspaces spanned by mode shape groups

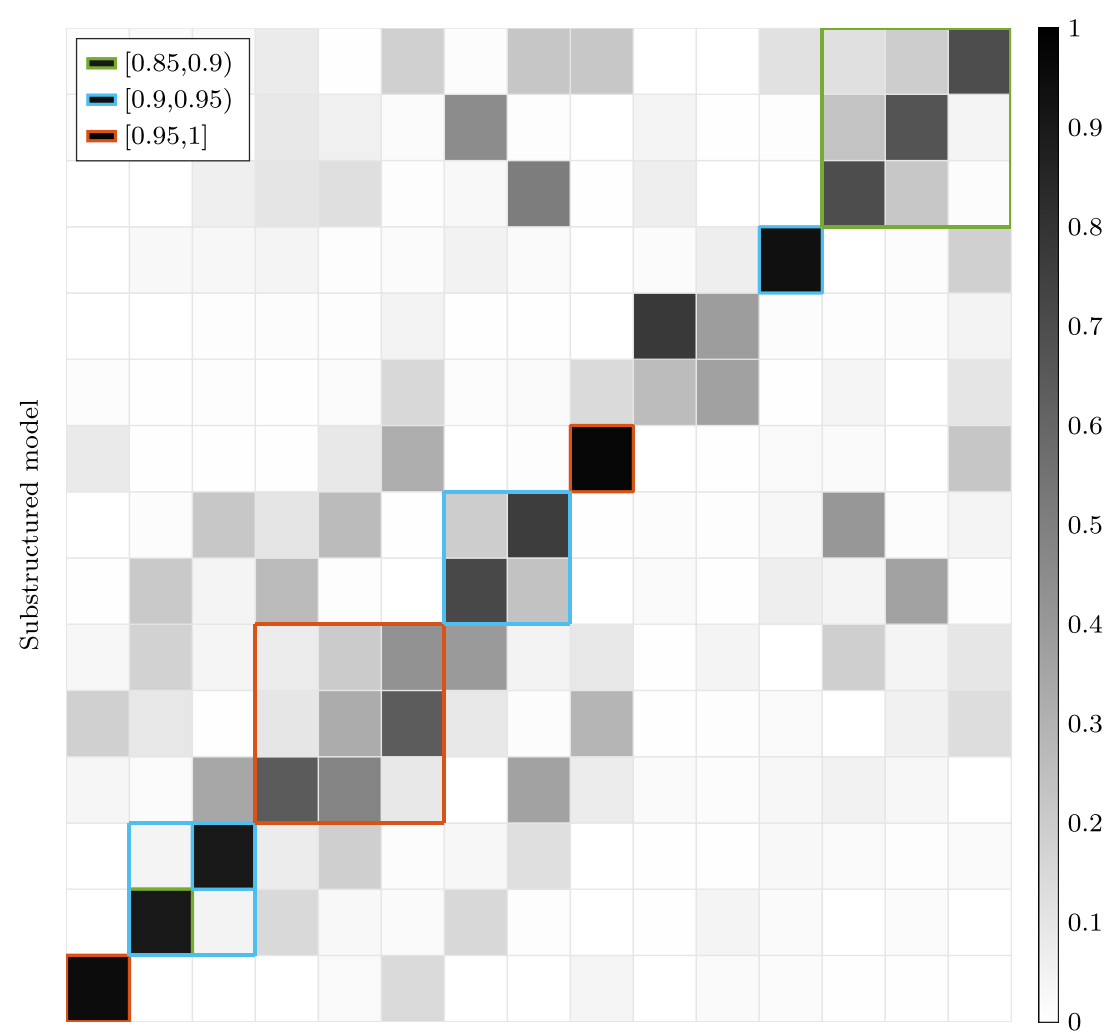

True model 
better than the nominal FE model for most modes, except for modes 1 and 6 . The same conclusion can be drawn from investigating the FRFs in Fig. 8. Therefore, using experimental models to build up the model of the assembled structure produces better results compared to the FE model in this case.

\section{Discussion}

In this section, possible sources of errors are identified and potential improvements are suggested. As mentioned above, passivity of the identified state-space models is crucial for successful coupling. In this work the method of Liljerehn and Abrahamsson [19] was used which enforces passivity for each mode individually. This requirement is potentially unnecessarily strict. Another method to constrain the passivity has been proposed by McKelvey and Moheimani [27] but was not considered here.

The first flexible mode of the transmission simulator is above $1700 \mathrm{~Hz}$. This is well above the frequency range of interest in this paper. Therefore, the transmission simulator is treated as rigid, yielding the condition number 1.9 of the mode shape matrix $\boldsymbol{\Phi}_{\mathrm{a}}^{\text {(ts) }}$ used. An attempt was made using also flexible modes of the transmission simulator but that resulted in higher errors in the modal parameters. Adding more modes to the transmission simulator representation increased the condition number. In order to lower the condition number, more sensors have to be included along with more modes. However, this approach is limited by the number of sensors available. The condition number is believed to be a possible, if difficult to evaluate, metric for the applicability of the transmission simulator technique [24]. However, other effects may also have influenced the results since the increase in the number was not substantial. Judging the quality of the transmission simulator modal matrix is still an open question. Here we used the condition number as metric and the results obtained with this transmission simulator model are promising. These results stem, however, from one application only and might not be universally valid.

A visual inspection of the motion of the hub of the three substructures and the hub itself (transmission simulator) can give valuable information about the fulfilment of the constraint equations, see [32]. If the constraint equations are completely fulfilled, no difference in motion should be present between the models. In the CMS method it was found that for low frequency flexible modes the motion of some sensors differed, which can stem either from the modal relaxation of the constraints or from measurement errors. This inspection is possible in CMS since the modal coordinates used for coupling can be transformed back to physical coordinates. Thus far, the same inspection cannot be performed with the state-space synthesis technique as in the transformation to modal coordinates, it is assumed that all modal coordinates are exactly equal. After coupling, the physical motion can no longer be deduced in a direct way.

Other possible sources of errors include the measurement set-up. The sensor locations on the hub were mounted on the brackets as this enables excitation and measurement in the same direction due to the plane surface. This choice entails measuring closer to the joints even though it is known that the bolted joints introduce nonlinearities [30]. Hence, such effects might become more apparent in the chosen experimental set-up. It was found that the stinger resonance has no influence in the frequency range of interest. Above $400 \mathrm{~Hz}$, pronounced differences in the measurements were found which could be due to resonances in the suspension or slight misalignments of the stinger, see $[34,35]$ for more details. In total eleven sensors, listed in Section "System Identification Methodology", turned out to be defective and were removed from the system identification procedure. Repeating the measurement to achieve a full set of measured points could possibly improve the substructuring results.

The identified models in this work are believed to replicate the measurements well enough, as indicated by the results. However, the absolute value of the error for the first mode in the receptance FRF (used for coupling) is larger than for the other modes due to larger displacements. The rather high error in the substructuring results for the first mode might be explained by this error since Liljerehn and Abrahamsson [18] found that the peak height of the identified FRF has a large influence on the substructuring results. The high damping errors could be a sign of an inappropriate damping model. The main source of dissipation is likely to be in the joints, which is not necessarily well represented by modal damping [31].

\section{Conclusion}

In this paper, the transmission simulator technique is developed for use with the state-space substructuring method. The interface is coupled using the MCFS technique. The method is applied to the SEM substructuring focus group's Ampair A600 wind turbine test-bed. Three blades are individually attached to the same hub and measured, resulting in data from which models are identified using system identification. These models are then coupled with two negative transmission simulators, that is negative representation of the FE model of the hub, to arrive at a system with one hub and three blades. Results from the coupled system are then compared to the measured fully assembled three bladed hub system by comparing FRFs and modal properties. Physical properties of the identified models used for substructuring, such as passivity 
and adherence to Newton's second law, are checked and enforced where necessary. The overall substructuring results correlate well with the true system, but the damping has been overestimated. Moreover, the experimental-analytical substructured model predicts the system's behaviour better than a nominal FE model of the full system.

The transmission simulator has successfully been applied to the state-space synthesis method, and it has been shown that CMS and state-space coupling produce the same results. The results may be improved further with better measurements, as the measurements are believed to be a source of errors.

\section{Compliance with Ethical Standards}

Conflict of interests On behalf of all authors, the corresponding author states that there is no conflict of interest.

Open Access This article is distributed under the terms of the Creative Commons Attribution 4.0 International License (http:// creativecommons.org/licenses/by/4.0/), which permits unrestricted use, distribution, and reproduction in any medium, provided you give appropriate credit to the original author(s) and the source, provide a link to the Creative Commons license, and indicate if changes were made.

\section{Appendix A: State-space Synthesis}

The derivation starts from a general state-space system. For a derivation of the state-space synthesis in the general formulation laid out by de Klerk et al. [16] see Gibanica [9] and Gibanica et al. [10], which also allows for synthesis of multiple substructures simultaneously. Here the states $\boldsymbol{x}^{(s)}$ are no longer directly related to physical coordinates but are arbitrary, linear combinations of the physical coordinates. However, the inputs and outputs are known and the input and output vectors can be partitioned in coupling $\mathrm{c}$ and body $\mathrm{b}$ inputs and outputs as

$\boldsymbol{y}^{(s)}=\left[\begin{array}{l}\boldsymbol{y}_{\mathrm{c}}^{(s)} \\ \boldsymbol{y}_{\mathrm{b}}^{(s)}\end{array}\right] \quad$ and $\quad \boldsymbol{u}^{(s)}=\left[\begin{array}{l}\boldsymbol{u}_{\mathrm{c}}^{(s)} \\ \boldsymbol{u}_{\mathrm{b}}^{(s)}\end{array}\right]$.

Then the state-space system can be written as

$$
\begin{aligned}
\dot{\boldsymbol{x}}^{(s)} & =\boldsymbol{A}^{(s)} \boldsymbol{x}^{(s)}+\boldsymbol{B}^{(s)} \boldsymbol{u}^{(s)} \\
& =\boldsymbol{A}^{(s)} \boldsymbol{x}^{(s)}+\left[\begin{array}{ll}
\boldsymbol{B}_{\mathrm{c}}^{(s)} & \boldsymbol{B}_{\mathrm{b}}^{(s)}
\end{array}\right] \boldsymbol{u}^{(s)} \\
\boldsymbol{y}^{(s)} & =\left[\begin{array}{l}
\boldsymbol{C}_{\mathrm{d}}^{(s)} \\
\boldsymbol{C}_{\mathrm{b}}^{(s)}
\end{array}\right] \boldsymbol{x}^{(s)} .
\end{aligned}
$$

The subscript $\mathrm{d}$ in $\boldsymbol{C}_{\mathrm{d}}$ stands for displacement at the coupling DOFs. For synthesis of two substructures, compatibility and force equilibrium must be enforced at the coupling DOFs. For DOFs co-oriented and numbered in the same order, the relation for input and response between the uncoupled substructures, (1) and (2), and coupled system can be written as

$\overline{\boldsymbol{u}}_{\mathrm{c}}=\left[\begin{array}{ll}\boldsymbol{I} & \boldsymbol{I}\end{array}\right]\left[\begin{array}{l}\boldsymbol{u}_{\mathrm{c}}^{(1)} \\ \boldsymbol{u}_{\mathrm{c}}^{(2)}\end{array}\right] \quad$ and $\quad\left[\begin{array}{l}\boldsymbol{y}_{\mathrm{c}}^{(1)} \\ \boldsymbol{y}_{\mathrm{c}}^{(2)}\end{array}\right]=\left[\begin{array}{l}\boldsymbol{I} \\ \boldsymbol{I}\end{array}\right] \overline{\boldsymbol{y}}_{\mathrm{c}}$.

Here $\overline{\boldsymbol{u}}_{\mathrm{c}} \in \mathbb{R}^{n_{\mathrm{c}} \times 1}$ and $\overline{\boldsymbol{y}}_{\mathrm{c}} \in \mathbb{R}^{n_{\mathrm{c}} \times 1}$ denote the coupled input and response, respectively, with $n_{\mathrm{c}}$ indicating the number of coupling DOFs. Before the systems can be coupled, the state vector $\boldsymbol{x}$ is transformed such that the coupling DOFs are represented by the displacement and velocity at the interface, $\boldsymbol{y}_{\mathrm{c}}^{(s)}$ and $\dot{y}_{\mathrm{c}}^{(s)}$. This transformation is defined as

$\tilde{\boldsymbol{x}}^{(s)}=\boldsymbol{T}^{(s)} \boldsymbol{x}(s)=\left[\begin{array}{c}\dot{\boldsymbol{y}}_{\mathrm{c}}^{(s)} \\ \boldsymbol{y}_{\mathrm{c}}^{(s)} \\ \boldsymbol{x}_{\mathrm{b}}^{(s)}\end{array}\right]$

and the new state-space representation, known as the coupling form, is defined as

$\tilde{\boldsymbol{A}}^{(s)} \triangleq \boldsymbol{T}^{(s)} \boldsymbol{A}^{(s)} \boldsymbol{T}^{(s)^{-1}}=\left[\begin{array}{ccc}\boldsymbol{A}_{\mathrm{vv}}^{(s)} & \boldsymbol{A}_{\mathrm{vd}}^{(s)} & \boldsymbol{A}_{\mathrm{vb}}^{(s)} \\ \boldsymbol{I}^{(s)} & \mathbf{0}^{(s)} & \mathbf{0}^{(s)} \\ \mathbf{0}^{(s)} & \boldsymbol{A}_{\mathrm{bd}}^{(s)} & \boldsymbol{A}_{\mathrm{bb}}^{(s)}\end{array}\right]$
$\tilde{\boldsymbol{B}}^{(s)} \triangleq \boldsymbol{T}^{(s)} \boldsymbol{B}^{(s)}=\left[\begin{array}{cc}\boldsymbol{B}_{\mathrm{vv}}^{(s)} & \boldsymbol{B}_{\mathrm{vb}}^{(s)} \\ \mathbf{0}^{(s)} & \mathbf{0}^{(s)} \\ \mathbf{0}^{(s)} & \boldsymbol{B}_{\mathrm{bb}}^{(s)}\end{array}\right]$
$\tilde{\boldsymbol{C}}^{(s)} \triangleq \boldsymbol{C}^{(s)} \boldsymbol{T}^{(s)}{ }^{-1}=\left[\begin{array}{lll}\mathbf{0}^{(s)} & \boldsymbol{I}^{(s)} & \mathbf{0}^{(s)} \\ \boldsymbol{C}_{\mathrm{bv}}^{(s)} & \boldsymbol{C}_{\mathrm{bd}}^{(s)} & \boldsymbol{C}_{\mathrm{bb}}^{(s)}\end{array}\right]$,

where the subscript $\mathrm{v}$ stands for velocity at the coupling DOFs. To couple two state-space models, equation (19) and equation (21) is used to arrive at the synthesised system

$$
\begin{aligned}
{\left[\begin{array}{c}
\ddot{\overline{\boldsymbol{y}}}_{\mathrm{c}} \\
\dot{\bar{y}}_{\mathrm{c}} \\
\dot{\boldsymbol{x}}_{\mathrm{b}}^{(1)} \\
\dot{\boldsymbol{x}}_{\mathrm{b}}^{(2)}
\end{array}\right]=} & {\left[\begin{array}{cccc}
\overline{\boldsymbol{A}}_{\mathrm{vv}} & \overline{\boldsymbol{A}}_{\mathrm{vd}} & \overline{\boldsymbol{A}}_{\mathrm{vb}}^{(1)} & \overline{\boldsymbol{A}}_{\mathrm{vb}}^{(2)} \\
\boldsymbol{I} & \mathbf{0} & \mathbf{0} & \mathbf{0} \\
\mathbf{0} & \boldsymbol{A}_{\mathrm{bd}}^{(1)} & \boldsymbol{A}_{\mathrm{bb}}^{(1)} & \mathbf{0} \\
\mathbf{0} & \boldsymbol{A}_{\mathrm{bd}}^{(2)} & \mathbf{0} & \boldsymbol{A}_{\mathrm{bb}}^{(2)}
\end{array}\right]\left[\begin{array}{c}
\dot{\overline{\boldsymbol{y}}}_{\mathrm{c}} \\
\overline{\boldsymbol{y}}_{\mathrm{c}} \\
\boldsymbol{x}_{\mathrm{b}}^{(1)} \\
\boldsymbol{x}_{\mathrm{b}}^{(2)}
\end{array}\right] } \\
+ & {\left[\begin{array}{ccc}
\overline{\boldsymbol{B}}_{\mathrm{vv}} & \overline{\boldsymbol{B}}_{\mathrm{vb}}^{(1)} & \overline{\boldsymbol{B}}_{\mathrm{vb}}^{(2)} \\
\mathbf{0} & \mathbf{0} & \mathbf{0} \\
\mathbf{0} & \boldsymbol{B}_{\mathrm{bb}}^{(1)} & \mathbf{0} \\
\mathbf{0} & \mathbf{0} & \boldsymbol{B}_{\mathrm{bb}}^{(2)}
\end{array}\right]\left[\begin{array}{c}
\overline{\boldsymbol{u}}_{\mathrm{c}} \\
\boldsymbol{u}_{\mathrm{b}}^{(1)} \\
\boldsymbol{u}_{\mathrm{b}}^{(2)}
\end{array}\right] } \\
{\left[\begin{array}{c}
\overline{\boldsymbol{y}}_{c} \\
\boldsymbol{y}_{\mathrm{b}}^{(1)} \\
\boldsymbol{y}_{\mathrm{b}}^{(2)}
\end{array}\right]=} & {\left[\begin{array}{cccc}
\mathbf{0} & \boldsymbol{I} & \mathbf{0} & \mathbf{0} \\
\boldsymbol{C}_{\mathrm{bv}}^{(1)} & \boldsymbol{C}_{\mathrm{bd}}^{(1)} & \boldsymbol{C}_{\mathrm{bb}}^{(1)} & \mathbf{0} \\
\boldsymbol{C}_{\mathrm{bv}}^{(2)} & \boldsymbol{C}_{\mathrm{bd}}^{(2)} & \mathbf{0} & \boldsymbol{C}_{\mathrm{bb}}^{(2)}
\end{array}\right]\left[\begin{array}{c}
\dot{\overline{\boldsymbol{y}}}_{\mathrm{c}} \\
\dot{\bar{y}}_{\mathrm{c}} \\
\boldsymbol{x}_{\mathrm{b}}^{(1)} \\
\boldsymbol{x}_{\mathrm{b}}^{(2)}
\end{array}\right] }
\end{aligned}
$$

with

$$
\begin{aligned}
\boldsymbol{K} & =\left(\boldsymbol{B}_{\mathrm{vv}}^{(1)}+\boldsymbol{B}_{\mathrm{vv}}^{(2)}\right)^{-1} \\
\overline{\boldsymbol{A}}_{\mathrm{vv}} & =\boldsymbol{B}_{\mathrm{vv}}^{(1)} \boldsymbol{K} \boldsymbol{A}_{\mathrm{vv}}^{(2)}+\boldsymbol{B}_{\mathrm{vv}}^{(2)} \boldsymbol{K} \boldsymbol{A}_{\mathrm{vv}}^{(1)} \\
\overline{\boldsymbol{A}}_{\mathrm{vd}} & =\boldsymbol{B}_{\mathrm{vv}}^{(1)} \boldsymbol{K} \boldsymbol{A}_{\mathrm{vd}}^{(2)}+\boldsymbol{B}_{\mathrm{vv}}^{(2)} \boldsymbol{K} \boldsymbol{A}_{\mathrm{vd}}^{(1)} \\
\overline{\boldsymbol{A}}_{\mathrm{vb}}^{(1)} & =\boldsymbol{B}_{\mathrm{vv}}^{(2)} \boldsymbol{K} \boldsymbol{A}_{\mathrm{vb}}^{(1)}
\end{aligned}
$$


$\overline{\boldsymbol{A}}_{\mathrm{vb}}^{(2)}=\boldsymbol{B}_{\mathrm{vv}}^{(1)} \boldsymbol{K} \boldsymbol{A}_{\mathrm{vb}}^{(2)}$

$\overline{\boldsymbol{B}}_{\mathrm{vv}}=\boldsymbol{B}_{\mathrm{vv}}^{(1)} \boldsymbol{K} \boldsymbol{B}_{\mathrm{vv}}^{(2)}$

$\overline{\boldsymbol{B}}_{\mathrm{vb}}^{(1)}=\boldsymbol{B}_{\mathrm{vv}}^{(2)} \boldsymbol{K} \boldsymbol{B}_{\mathrm{vb}}^{(1)}$

$\overline{\boldsymbol{B}}_{\mathrm{vb}}^{(2)}=\boldsymbol{B}_{\mathrm{vv}}^{(1)} \boldsymbol{K} \boldsymbol{B}_{\mathrm{vb}}^{(2)}$.

The transformation matrix $\boldsymbol{T}^{(s)}$ is found as follows. First, the matrix

$\boldsymbol{T}_{0}^{(s)}=\left[\begin{array}{c}\boldsymbol{C}_{\mathrm{d}}^{(s)} \boldsymbol{A}^{(s)} \\ \boldsymbol{C}_{\mathrm{d}}^{(s)} \\ \boldsymbol{T}_{0,3}^{(s)}\end{array}\right]$

is defined, with $\boldsymbol{T}_{0,3}^{(s)}$ being an arbitrary nullspace of $\boldsymbol{B}_{\mathrm{c}}^{(s)}$, $\boldsymbol{T}_{0,3}^{(s)} \boldsymbol{B}_{\mathrm{c}}^{(s)}=\mathbf{0}$, such that $\boldsymbol{T}_{0}^{(s)}$ is non-singular. The final transformation is given by

$\boldsymbol{T}^{(s)}=\left[\begin{array}{c}\boldsymbol{C}_{\mathrm{d}}^{(s)} \boldsymbol{A}^{(s)} \\ \boldsymbol{C}_{\mathrm{d}}^{(s)} \\ \boldsymbol{T}_{0,3}^{(s)}\left(\boldsymbol{I}^{(s)}-\boldsymbol{A}^{(s)} \boldsymbol{Z}_{0,1}^{(s)} \boldsymbol{C}_{d}^{(s)}\right)\end{array}\right]$

where

$\boldsymbol{Z}_{0}^{(s)}=\boldsymbol{T}_{0}^{(s)^{-1}} \triangleq\left[\begin{array}{lll}\boldsymbol{Z}_{0,1}^{(s)} & \boldsymbol{Z}_{0,2}^{(s)} & \boldsymbol{Z}_{0,3}^{(s)}\end{array}\right]$.

Here the identity matrix $\boldsymbol{I}^{(s)} \in \mathbb{R}^{n^{(s)} \times n^{(s)}}$, the transformation matrix $\boldsymbol{T}^{(s)} \in \mathbb{R}^{n^{(s)} \times n^{(s)}}$, partition $\boldsymbol{T}_{0,3}^{(s)} \in \mathbb{R}^{n^{(s)}-2 n_{\mathrm{c}} \times n^{(s)}}$ and $Z_{0,1}^{(s)} \in \mathbb{R}^{n^{(s)} \times n_{\mathrm{c}}}$.

Note that important prerequisites exist for using this method. The systems used for the synthesis need to be passive and physically consistent which must be ensured during the system identification process. In addition, $\boldsymbol{B}_{\mathrm{c}}^{(s)}$ and $\boldsymbol{C}_{\mathrm{d}}^{(s)}$ must have full row rank and full column rank, respectively. The matrix $\boldsymbol{B}_{\mathrm{vv}}^{(s)}=\boldsymbol{C}_{d}^{(s)} \boldsymbol{A}^{(s)} \boldsymbol{B}_{\mathrm{c}}^{(s)}$ must be full rank to compute its inverse which corresponds to the interface inertia [38]. In [9, 10] Gibanica revealed numerical difficulties of the state-space approach applied to a complex structure, e.g. wind turbines, that stem from the choice of the null space in the coupling form transformation. As a remedy, all state-space systems will be diagonalised in this paper before the transformation is performed.

\section{Appendix B: Transmission Simulator}

In this section, the concept of the transmission simulator will be explained and decoupling of structures will be derived for the CMS method following the paper of Allen et al. [4]. The coupling constraint, modal constraints for fixture and subsystem (MCFS), will also be explained.

In the modal domain, decoupling is achieved by adding a system with negative modal mass, damping, and stiffness. If the structure to be subtracted is denoted transmission simulator (ts) and decoupled from the total system (tot), the uncoupled block-diagonal form of both systems with $k_{\mathrm{ts}}$ transmission simulator systems is

$$
\begin{aligned}
& {\left[\begin{array}{cc}
\boldsymbol{M}_{\mathrm{mod}}^{(\mathrm{tot})} & \mathbf{0} \\
\mathbf{0} & -k_{\mathrm{ts}} \boldsymbol{M}_{\mathrm{mod}}^{(\mathrm{ts})}
\end{array}\right]\left[\begin{array}{l}
\ddot{\boldsymbol{\eta}}^{(\mathrm{tot})} \\
\ddot{\boldsymbol{\eta}}^{(\mathrm{ts})}
\end{array}\right]} \\
& +\left[\begin{array}{cc}
\boldsymbol{V}_{\text {mod }}^{(\mathrm{tot})} & \mathbf{0} \\
\mathbf{0} & -k_{\mathrm{ts}} \boldsymbol{V}_{\mathrm{mod}}^{(\mathrm{ts})}
\end{array}\right]\left[\begin{array}{c}
\dot{\boldsymbol{\eta}}^{(\mathrm{tot})} \\
\dot{\boldsymbol{\eta}}^{(\mathrm{ts})}
\end{array}\right] \\
& +\left[\begin{array}{cc}
\boldsymbol{K}_{\mathrm{mod}}^{(\mathrm{tot})} & \mathbf{0} \\
\mathbf{0} & -k_{\mathrm{ts}} \boldsymbol{K}_{\mathrm{modd}}^{(\mathrm{ts})}
\end{array}\right]\left[\begin{array}{c}
\boldsymbol{\eta}^{(\mathrm{tot})} \\
\boldsymbol{\eta}^{(\mathrm{ts})}
\end{array}\right] \\
& =\left[\begin{array}{c}
\boldsymbol{f}_{\text {mod }}^{\text {(tot) }} \\
\boldsymbol{f}_{\mathrm{mod}}^{\text {(ts) }}
\end{array}\right] .
\end{aligned}
$$

To circumvent the problem where the observation of the connection points' motion based on the measurement points is insufficient, the MCFS method can be used. In the MCFS method the coupling is enforced by constraining the displacement of all points on the transmission simulator of the total system $\boldsymbol{q}_{\mathrm{a}}^{\text {(tot) }} \in \mathbb{R}^{n_{\mathrm{a}} \times 1}$ to be equal to the motion of the analytical transmission simulator model $\boldsymbol{q}_{\mathrm{a}}^{(\mathrm{ts})} \in \mathbb{R}^{n_{\mathrm{a}} \times 1}$ which is expressed as

$\boldsymbol{q}_{\mathrm{a}}^{(\mathrm{ts})}=\boldsymbol{q}_{\mathrm{a}}^{(\mathrm{tot})}$.

Again, the subscript a denotes the points on the transmission simulator that have been measured ( $n_{\mathrm{a}}$ in number) in the total system, i.e. $\boldsymbol{q}_{\mathrm{a}}^{\text {(tot) }}$. If more measurement points than modes exist, some coupling conditions will be redundant and may cause problems like lock-down behaviour. Therefore, it is necessary to relax the constraints and fulfil them in a least squares sense. This is done in terms of the transmission simulator modal matrix $\boldsymbol{\Phi}_{\mathrm{a}}^{(\mathrm{ts})} \in \mathbb{R}^{n_{\mathrm{a}} \times m_{\mathrm{ts}}}$, where $m_{\mathrm{ts}}$ denote the number of modes selected from the transmission simulator model. The pseudo-inverse of the modal matrix is multiplied to equation (28) such that

$\boldsymbol{\Phi}_{\mathrm{a}}^{(\mathrm{ts})^{+}} \boldsymbol{q}_{\mathrm{a}}^{(\mathrm{ts})}=\boldsymbol{\Phi}_{\mathrm{a}}^{(\mathrm{ts})^{+}} \boldsymbol{q}_{\mathrm{a}}^{(\mathrm{tot})}$.

Note that the number of constraints equals the number of modes in the representation of the transmission simulator and that the mode shapes in $\boldsymbol{\Phi}_{\mathrm{a}}^{(\mathrm{ts})}$ must be linearly independent.

\section{References}

1. AbraDAQ: https://github.com/mgcth/abraDAQ. (visited on 201805-24)

2. Allemang RJ, Brown DL (1982) A correlation coefficient for modal vector analysis. In: Proceedings of the 1st IMAC

3. Allen MS, Kammer DC, Mayes RL (2012) Metrics for diagnosing negative mass and stiffness when uncoupling experimental and analytical substructures. J Sound Vib 331(25):5435-5448

4. Allen MS, Mayes RL, Bergman EJ (2010) Experimental modal substructuring to couple and uncouple substructures with flexible fixtures and multi-point connections. J Sound Vib 329(23):48914906 
5. Bathe KJ (1982) Finite element procedures in engineering analysis. Prentice Hall, Englewood Cliffs

6. Brincker R, Ventura C (2015) Introduction to operational modal analysis. Wiley, Chichester

7. D'Ambrogio W, Fregolent A (2012) Direct hybrid formulation for substructure decoupling. In: Topics in experimental dynamics substructuring and wind turbine dynamics, volume 2 , conference proceedings of the society for experimental mechanics series. Springer, New York, pp 89-107

8. Ewins DJ (2000) Modal testing: theory, practice and application, 2nd. Wiley-Blackwell, Philadelphia

9. Gibanica M (2013) Experimental-analytical dynamic substructuring: a state-space approach. Master's Thesis, Chalmers University of Technology

10. Gibanica M, Johansson AT, Liljerehn A, Sjövall P, Abrahamsson $T$ (2014) Experimental - analytical dynamic substructuring of ampair testbed: a state-space approach. In: Allen M, Mayes R, Rixen D (eds) Dynamics of coupled structures, volume 1, conference proceedings of the society for experimental mechanics series. Springer International Publishing, pp 1-14

11. Gibanica M, Johansson AT, Rahrovani S, Khorsand M, Abrahamsson T (2014) Spread in modal data obtained from wind turbine blade testing. In: Mayes R, Rixen D, Allen M (eds) Topics in experimental dynamic substructuring, volume 2 , conference proceedings of the society for experimental mechanics series. Springer, New York, pp 207-215

12. Harvie J, Avitabile P (2012) Comparison of some wind turbine blade tests in various configurations. In: Topics in experimental dynamics substructuring and wind turbine dynamics, vol 2 . Springer, New York, pp 73-79

13. Jetmundsen B, Bielawa RL, Flannelly WG (1988) Generalized frequency domain substructure synthesis. J Am Helicopter Soc 33(1):55-64

14. Johansson AT, Lindholm CJ, Vakilzadeh K, Abrahamsson T (2014) Modeling and calibration of small-Scale wind turbine blade. In: Topics in experimental dynamic substructuring, vol 2. Springer, New York, pp 51-58

15. Kammer DC, Allen MS, Mayes RL (2014) Formulation of a craig-Bampton experimental substructure using a transmission simulator. In: Topics in experimental dynamic substructuring, volume 2, conference proceedings of the society for experimental mechanics series. Springer, New York, pp 321-331

16. de Klerk D, Rixen DJ, Voormeeren SN (2008) General framework for dynamic substructuring: history, review and classification of techniques. AIAA J 46(5):1169-1181

17. Liljerehn A (2016) Machine tool dynamics - a constrained state-space substructuring approach. Doctoral thesis, Chalmers University of Technology

18. Liljerehn A, Abrahamsson T (2012) Experimental analytical substructure model sensitivity analysis for cutting machine chatter prediction. In: Topics in experimental dynamics substructuring and wind turbine dynamics, vol 2. Springer, New York, pp 11-20

19. Liljerehn A, Abrahamsson T (2014) Dynamic sub-structuring with passive state-space components. In: Proceedings of the 26th ISMA, pp 3879-3890. Leuven

20. Ljung L (1999) System identification: theory for the user, 2nd. Upper Saddle River, Prentice Hall

21. Maia NMM, Silva JMM (eds) (1997) Theoretical and experimental modal analysis, 1st. Research Studies Press, Baldock

22. Mayes RL (2012) An introduction to the SEM substructures focus group test bed - the ampair 600 wind turbine. In: Topics in experimental dynamics substructuring and wind turbine dynamics, vol 2. Springer, New York, pp 61-70
23. Mayes RL, Allen MS, Kammer DC (2012) Eliminating indefinite mass matrices with the transmission simulator method of substructuring. In: Topics in experimental dynamics substructuring and wind turbine dynamics, volume 2 , conference proceedings of the society for experimental mechanics series. Springer, New York, pp 21-31

24. Mayes RL, Arviso M (2010) Design studies for the transmission simulator method of experimental dynamic substructuring. In: Proceedings of the 24th ISMA, pp 1929-1938. Leuven

25. Mayes RL, Hunter PS, Simmermacher TW, Allen MS (2008) Combining experimental and analytical substructures with multiple connections. In: Proceedings of the 26th IMAC

26. McKelvey T, Akcay H, Ljung L (1996) Subspace-based multivariable system identification from frequency response data. IEEE Trans Autom Control 41(7):960-979

27. McKelvey T, Reza Moheimani SO (2005) Estimation of phase constrained MIMO transfer functions with application to flexible structures with mixed collocated and non-collocated actuators and sensors. IFAC Proc 38(1):219-224

28. van Overschee P, de Moor BL (1996) Subspace identification for linear systems, theory - implementation - applications, reprint of the original, 1st. Springer, Boston

29. Reddy JN (2007) An introduction to continuum mechanics. Cambridge University Press, New York

30. Reuss P, Kruse S, Peter S, Morlock F, Gaul L (2014) Identification of nonlinear joint characteristic in dynamic substructuring. In: Topics in experimental dynamic substructuring, vol 2. Springer, New York, pp 27-36

31. Roettgen DR, Mayes RL (2015) Ampair 600 wind turbine threebladed assembly substructuring using the transmission simulator method. In: Dynamics of coupled structures, vol 4. Springer, Cham, pp 111-123

32. Rohe DP, Mayes RL (2014) Coupling of a bladed hub to the tower of the ampair 600 wind turbine using the transmission simulator method. In: Topics in experimental dynamic substructuring, vol 2. Springer, New York, pp 193-206

33. Sandia National Laboratories: Substructuring Testbed Assembly Instructions. Tech rep. (2012)

34. Scheel M (2015) State-space experimental-analytical dynamic substructuring using the transmission simulator. Master's Thesis, Chalmers University of Technology

35. Scheel M, Johansson AT (2016) State-space substructuring with transmission simulator. In: Dynamics of coupled structures, vol 4. Springer, Cham, pp 91-103

36. van der Seijs MV (2016) Experimental dynamic substructuring: Analysis and design strategies for vehicle development. Doctoral thesis, Delft University of Technology, Delft

37. van der Seijs MV, de Klerk D, Rixen DJ, Rahimi S (2014) Validation of current state frequency based substructuring technology for the characterisation of steering gear-vehicle interaction. In: Topics in experimental dynamic substructuring, volume 2, conference proceedings of the society for experimental mechanics series. Springer, New York, pp 253-266

38. Sjövall P (2004) Component synthesis and identification in structural dynamics. Licentiate thesis, Chalmers University of Technology

39. Sjövall P, Abrahamsson T (2007) Component system identification and state-space model synthesis. Mech Syst Signal Process 21(7):2697-2714

40. Sjövall P, McKelvey T, Abrahamsson T (2006) Constrained state-space system identification with application to structural dynamics. Automatica 42(9):1539-1546 
41. Su TJ, Juang JN (1994) Substructure system identification and synthesis. J Guid Control Dyn 17(5):1087-1095

42. Verhaegen M, Verdult V (2007) Filtering and system identification: a least squares approach, 1st. Cambridge University Press, Cambridge
43. Voormeeren SN (2012) Dynamic substructuring methodologies for integrated dynamic analysis of wind turbines. Ph.D. thesis, Delft University of Technology, Delft

Publisher's Note Springer Nature remains neutral with regard to jurisdictional claims in published maps and institutional affiliations. 